\title{
Test on the effectiveness of the sum over paths approach in favoring the construction of an integrated knowledge of quantum physics in high school
}

\author{
Massimiliano Malgieri, ${ }^{1,}{ }^{*}$ Pasquale Onorato, ${ }^{2}$ and Anna De Ambrosis ${ }^{1}$ \\ ${ }^{1}$ Department of Physics, University of Pavia, Via Bassi 6, 27100 Pavia, Italy \\ ${ }^{2}$ Department of Physics, University of Trento, Via Sommarive 14, I-38123 Povo, Trentino, Italy \\ (Received 13 April 2016; published 3 January 2017; publisher error corrected 19 January 2017)
}

\begin{abstract}
In this paper we present the results of a research-based teaching-learning sequence on introductory quantum physics based on Feynman's sum over paths approach in the Italian high school. Our study focuses on students' understanding of two founding ideas of quantum physics, wave particle duality and the uncertainty principle. In view of recent research reporting the fragmentation of students' mental models of quantum concepts after initial instruction, we collected and analyzed data using the assessment tools provided by knowledge integration theory. Our results on the group of $n=14$ students who performed the final test indicate that the functional explanation of wave particle duality provided by the sum over paths approach may be effective in leading students to build consistent mental models of quantum objects, and in providing them with a unified perspective on both the photon and the electron. Results on the uncertainty principle are less clear cut, as the improvements over traditional instruction appear less significant. Given the low number of students in the sample, this work should be interpreted as a case study, and we do not attempt to draw definitive conclusions. However, our study suggests that (i) the sum over paths approach may deserve more attention from researchers and educators as a possible route to introduce basic concepts of quantum physics in high school, and (ii) more research should be focused not only on the correctness of students' mental models on individual concepts, but also on the ability of students to connect different ideas and experiments related to quantum theory in an organized whole.
\end{abstract}

DOI: 10.1103/PhysRevPhysEducRes.13.010101

\section{INTRODUCTION}

A major goal of physics education has always been to provide students with an integrated, unifying perspective of disciplinary knowledge, through the use of far-reaching models and concepts, contributing to avoiding fragmentation of knowledge. When considering the problem of introducing elements of quantum physics in secondary school, the objective of providing a unifying framework appears all the more urgent. Physics education research has often highlighted that, even in undergraduate introductory courses, students tend to build fragmented mental models of basic quantum concepts, such as wave particle duality and the uncertainty principle.

The "sum over paths" approach to the teaching of quantum physics based on Feynman's path integral formulation [1-3] was first introduced by Taylor and coauthors [4] in the context of a course for high school science teachers at Montana State University. Several have adopted and expanded the approach in introductory

\footnotetext{
*Corresponding author. massimiliano.malgieri01@universitadipavia.it

Published by the American Physical Society under the terms of the Creative Commons Attribution 3.0 License. Further distribution of this work must maintain attribution to the author(s) and the published article's title, journal citation, and DOI.
}

undergraduate courses [5], teacher preparation courses [6,7], and directly with high school students [8]. In Great Britain, the ambitious large-scale project "Advancing Physics AS" [9,10] of an A-level high school course in physics produced by the Institute of Physics treated quantum physics using the sum over paths approach.

The sum over paths approach has several characteristics which make it attractive in the design of an introductory sequence aimed at providing students with an integrated perspective on quantum physics. First, Feynman's approach offers students a very clear and unambiguous route to building an adequate mental model of one of its most profound mysteries, wave particle duality. It allows one to identify a central difference between classical and quantum physics in the rule for computing the probability of an event which can happen in several alternative, undistinguishable ways; and by doing so it permits one to construct a language capable of discussing several modern experiments in quantum optics. It makes the classical limit completely transparent, allowing an easy and convincing derivation of the classical laws from the rules valid for quantum objects. Finally, it requires little advanced mathematics, and uses a very simple formal language, allowing students to concentrate on the conceptual aspects of the theory. At its heart, such a possibility is due to the fact that, rather than finding solutions to the 
Schrödinger equation, Feynman's method constructs the Green's function for the same equation, representing it as a sum of complex amplitudes computed over all possible paths. In educational practice, complex amplitudes associated to paths can be represented and added up as vectors or "little arrows" [2].

Despite having appeared in the physics education community more than fifteen years ago, and having been adopted in a relatively large number of settings, no structured evaluation of the effectiveness of the approach with high school students has been published.

With this paper, we aim at contributing to fill such a gap, by presenting the results of an experimentation carried out in the Italian secondary school. In particular, our study concentrates on two aspects: (i) what kind of mental models students build of basic quantum concepts, and in particular of wave particle duality and the uncertainty principle, when they are presented to them using the sum over paths perspective; and (ii) whether Feynman's approach can help teachers in the task of providing students with an integrated model of quantum physics, rather than with seemingly disconnected models valid in the context of one experiment only.

\section{A. Research on student's conceptual difficulties in basic quantum physics}

A large amount of literature exists on students' difficulties in learning basic quantum physics. Many of these difficulties can be classified as conceptual, in the sense that they are related to students' intuitive, qualitative reasoning schemes and mental models. Difficulties of this kind often appear even when students have successfully acquired procedural abilities, so that in some cases they may be able to solve traditional problems requiring the application of formulas, but not to answer to basic, qualitative questions on the behavior of quantum objects and systems [11]. Many studies have reported that student's alternative models are resistant to instruction, as very little advancement may take place in conceptual understanding of basic concepts such as wave particle duality and the uncertainty principle while progressing with the study of quantum physics $[12,13]$.

A recurring characteristic of alternative models produced by students on different conceptual issues is that they appear to be constructed by trying to progressively incorporate quantum elements upon a pre-existing classical framework. Hybrid conceptions constructed in this way introduce some quantum elements while retaining essential features of the classical world view [14]. Students' models after introductory instruction have been found to be fragmented, made up mainly of memorized facts, and consistent only in the context of an individual phenomenon or experiment [15-16].

In the rest of this section, in accord with the aims of this study, we focus only on the existing literature on students' difficulties on wave particle duality and the uncertainty principle.

\section{Wave particle duality}

The concept of wave particle duality is well known to be problematic in the teaching of quantum physics, and students' difficulties and alternate models are well studied. In a large $(n=236)$ study with final-year Norwegian high school students, Olsen [17] finds a very high incidence of hybrid models of wave particle duality based on classical concepts, and concludes that students' understanding of the idea following high school instruction is severely limited. One of the most important findings of the study is that students do not build a unified model, or even similar models, for photons and electrons: in fact, $59 \%$ of students interpret electrons as being basically classical particles, while $77 \%$ attribute a dual nature to photons, although in most cases they cannot correctly explain how this duality manifests itself. Others $[18,19]$ have confirmed this finding, reporting it also at different levels of instruction.

A common alternative conception that arises in students when they try to accommodate the idea of wave particle duality into their preexisting classical framework consists of depicting classical particles as following sinusoidal trajectories [17,20,21]. A variant of this mental model consists of imagining quantum objects undergoing random oscillations reminiscent of Brownian motion [13]. Students also build realistic mental models based on the wave ontology, such as of the matter-wave [16,19], or "energy lump" [18] categories. Instruction on the concept of wave particle duality often involves discussion of the two slit experiment, and in this context students may form the synthetic conception that interference is due to particleparticle interactions [21]. Again, electrons have been found to more strongly evocate this model than photons [19], partly because of their charged nature.

The study of Olsen [17] cited previously implies that the traditional approach to teaching quantum physics in secondary school may be inefficient in forming a consistent model of wave particle duality. In the sample investigated, the answers classified as genuine duality were only $0.4 \%$ for photons and $2.5 \%$ for electrons. Several have reported that the typical university approach is also only partially effective in this respect. Mannila et al. [13] performed a study with $n_{1}=8$ undergraduate students aiming to a degree as physics teachers and $n_{2}=21$ undergraduates in general physics, attending an intermediate level course in quantum physics. They focused on students' conceptions of the photon, and found that in both cases less than half of student's answers were categorized in the two acceptable classes of "dual description" and "statistical description" of the photon. Ayene et al. [19] performed a study on the students' mental model of wave particle duality and the uncertainty principle with $n=25$ Ethiopian undergraduate physics students. They found that a vast majority of these 
students adopted a classical or quasiclassical model of duality, while only in a small number of cases the answers of students were categorized as providing a quantum description.

\section{The uncertainty principle}

In its modern understanding, the uncertainty principle (often referred to as Heisenberg's uncertainty principle) is a property connected to the preparation of a quantum state, and it asserts that it is impossible to prepare in any way a state of a quantum object such that the indeterminacies in complementary variables violate the Heisenberg relation (e.g., $\Delta x \Delta p_{x} \geq \hbar / 2$ ). Those who investigated students' difficulties on the uncertainty principle $[15,19,22,23]$ identified two basic categories of alternate models constructed by students:

(a) Students may think of the principle as expressing an experimental limitation, i.e., that due to unavoidable noise, or excessive smallness of the quantities being measured, one cannot obtain measurements that are more accurate than a certain limit $[15,19,22]$. In this case, often students may also fail to recognize, or underestimate, the significance of the principle being formulated in terms of complementary variables; for example, they may think that neither position nor momentum can be measured beyond a certain precision [24].

(b) Students may think of uncertainty as a perturbation caused by measurement [19,22] reproducing, consciously or less, elements of the historical interpretation of the principle given by Heisenberg in his thought experiment of a single-photon microscope.

Some [14] consider the mental model (b) above as acceptable at the high school level. However, in the present study, in accordance with the most recent literature on the subject, we only consider as completely acceptable the model expressing a true intrinsic indeterminacy.

Alternate depictions of uncertainty are reportedly very common at the undergraduate level. For example, in the study by Ayene et al. [19] with $n=25$ Ethiopian undergraduates already mentioned in the previous section, only three students referred to the uncertainty principle as expressing intrinsic indeterminacy, while the others either adopted Heisenberg's measurement disturbance interpretation or thought of the principle as referring to some form of experimental error with respect to a "true" value. Müller and Wiesner [22] performed a similar study on a cohort of $n=37$ 3rd to 5 th year German perspective teachers, most of which had attended some quantum mechanics lecture at university. In this case, about half the students provided an acceptable answer, while the rest adopted one of the two interpretations (a) and (b) described above.

Among the few studies performed in high school, Ref. [23] is especially significant. Here, Müller and Wiesner compare the results of two groups of German
Gymnasiun students: the control group $\left(n_{1}=35\right)$ is introduced to the subject matter using the ordinary high school curriculum and textbook, while the experimental group $\left(n_{2} \approx 60\right)$ attends a research-based course of their own design. The authors do not discern between different alternative models of uncertainty, but only require students to rate the statement "In principle, quantum objects can simultaneously possess position and momentum." on a scale of 1 to 5 from "strongly agree" to "strongly disagree." In the control group, students disagreeing with the statement (marks 4 and 5) are about one-third of the sample. Students of the experimental group obtain much better results with a vast majority of students (about $80 \%$ ) giving 4 and 5 marks. It must be noted that three classes out of five in their experimental sample are made up of students with a special interest in physics, who are given a significantly more advanced course taught for 5 hours a week. Despite such limitations, Ref. [23] shows that the adoption of research-based methods and contents allows for vast improvements in students' understanding of Heisenberg's principle with respect to traditional teaching strategies.

\section{A note on the comparability of results obtained with different methods}

In our article we will use some previous works, and, in particular, those of Olsen [17] and of Müller and Wiesner [23], as a general reference for the expected level of understanding of wave particle duality and the uncertainty principle, respectively, in high school. It is clear that, strictly speaking, any detailed comparison of percentages is invalid, even setting aside the issue of the smallness of our sample, because the methods used are not identical. The procedure used by Olsen is actually similar to ours, in that student's explanations of wave particle duality are grouped in categories, and a definition as to what should be intended with a "genuinely quantum" explanation is provided. Müller and Wiesner, in assessing understanding of the uncertainty principle as genuine quantum indeterminacy, use a different approach based on similar premises, as described in the previous section. But having made all the necessary clarifications, the fact remains that most studies on conceptual understanding following initial instruction with traditional methods, both in high school and at the undergraduate level, while using different methods, highlight the same general trend lines in the results. It is in the spirit of taking these trend lines as a reference, that our comparisons will be made.

\section{B. Theoretical framework}

\section{The teaching of quantum physics as a problem of conceptual change}

Several $[25,26]$ have considered the problem of teaching quantum physics from the perspective of conceptual change. In particular, similarly to Ref. [25] we adopted 
the "framework theory" approach [27], deeming it as the most relevant to interpret the problems connected to introductory instruction in quantum physics. According to Vosniadou and co-workers, conceptual change has to be intended as the gradual replacement of a preexisting explanatory framework with another which is being acquired through instruction, a process which requires the learner to reassign objects to new ontological categories. The process often involves, and is facilitated by, metacognitive and metatheoretical reflection about the nature of the students' own entrenched presuppositions, and the structure of the new framework. While such process is taking place, the two frameworks may coexist for a long time, forming a dynamical system in constant development. As learners acquire information incompatible with their existing explanatory framework, they may try to subsume such information into categories that are acceptable for the old theory, reorganizing their knowledge in "synthetic" conceptions or models, which are forms of hybridization between the two frameworks. Synthetic models have some explanatory power for the student, as they allow them to account for phenomena incompatible with their preexisting beliefs, but often are consistent only within a limited domain. Indeed, we previously reported on several cases of students spontaneously formulating hybrid, synthetic classical-quantum models at intermediate stages of their learning process [28], and many other examples are highlighted in the literature.

Framework theory provides several suggestions for designing learning environments able to promote successful conceptual change. Many of these suggestions, which are efficiently recapitulated in Ref. [29], directly influenced the design of our teaching-learning sequence.

\section{Assessing integrated knowledge of quantum theory}

Several have reported that the mental models students develop in response to initial instruction in quantum physics are fragmented, and have only a limited consistence in restricted contexts. This is in accord with the point of view of framework theory, as synthetic conceptions obtained by embedding elements of a new paradigm in a previously existing, internally self-consistent one, allow the learner to reconcile some phenomena with their previous beliefs, while leaving unfilled "gaps" and emerging inconsistencies in the overall picture [30]. It follows that a significant test to decide whether conceptual change has happened in a particular learner may be to evaluate whether their ideas are robustly integrated in a connected and globally consistent network, for example, whether they are able to link different phenomena as examples of the same principle or law, or to analyze different experimental setups using the same conceptual tools.

In the science education literature, efficient assessment methods focused on the above objectives have been provided by knowledge integration theory [31,32]. From the knowledge integration perspective, science learning occurs when students are solicited to articulate and verbalize their ideas about the curriculum topic, add new normative ideas to their repertory, develop scientific criteria to distinguish between ideas, and form a more coherent view of science as a result of integrating various scientific ideas. A signature mark of the knowledge integration perspective is the emphasis on the importance of internal coherence in students' scientific frameworks, and in the connections among their ideas [33]. The resonances and connections between the model of conceptual change brought forward by framework theory and the perspective of knowledge integration are well established theoretically [34,35] and have been exploited by others [36,37].

A major success of knowledge integration theory has been the development of a general method for assessing students' successful learning of complex science topics, based on open response questions ("Knowledge Integration assessment tasks"), in which students are asked to explain scientific phenomena or facts producing an organized scientific discourse that links to other principles, laws, or phenomena. The answers to such items are graded using semistandardized scoring rubrics ("Knowledge Integration rubrics", or KI-rubric), which do not dichotomously characterize the answer as correct or incorrect, but evaluate, on a 5 or 6 grade scale, the number and/or the consistency and validity of the links to other scientific ideas that each student can produce in support to their answer. The method of KI assessment tasks and scoring rubrics has been shown to exhibit satisfactory psychometric properties, and to yield significantly higher discrimination indexes with respect to dichotomous grading scales [35].

\section{A teaching-learning sequence on basic quantum physics based on the sum over paths approach}

The teaching-learning sequence used in high school is the result of a two-year work of design and refinement, in which a first version was tested with student teachers [7]. Based on the results of the first test, the sequence was refined in preparation for a second test with student teachers [38] and the test in high school. The salient characteristics of our teaching-learning sequence (TLS) can be schematically described as follows:

- The sequence does not follow the historical development of quantum theory, but rather presents both historical evidence (e.g., the photoelectric and Compton effects) and modern experiments that are conveniently described using the sum over paths approach and carry a high conceptual significance (e.g., the two slit interference setup with one quantum object at a time, the Mach-Zehnder interferometer with individual photons, the Zhou-Wang-Mandel experiment [39]). One of the main reasons for departing from a historical approach is that for some concepts the currently accepted interpretation is significantly different from 
what it was in the first years after the formalization of quantum theory, having evolved in response to new evidence and emerging problems. In several cases, the results of modern experiments can be used to sharply counter the ingenuous predictions of students based on hybrid models, providing useful sources of cognitive conflicts at key points in the sequence.

- The sequence starts introducing the photon and the quantum phenomenology of light, aiming at presenting first a consistent model of photon behavior with all its quantum properties, highlighted by selected experiments in quantum optics. Then, the formalism is extended to massive particles, using the almost perfect analogy between the sum over paths formulations for the two types of quantum objects. The generality of the model is stressed by discussing similar or identical experimental setups realized using either photons or massive particles (e.g., the Young's experiment, single slit diffraction).

- The presentation avoids vague dualistic descriptions of quantum objects, and exploits the functional model of duality provided by the language of Feynman's approach. In the sum over paths perspective, in fact, the model only contains pointlike quantum objects, which simultaneously explore all possible paths; the idea of "wave particle duality" consists of the following two points: (i) quantum objects are always detected as localized entities, but their probability of detection is given by the quantum probability rule, which is responsible for the emergence of wavelike interference phenomena; (ii) if information is acquired on the system through a "which way" measurement, then the possible paths available to the system for a given outcome are reduced in number, and interference is lost; or, equivalently, the paths are no longer indistinguishable, so that the classical probability rule applies. The Zhou-Wang-Mandel and Mach-Zehnder experimental setups play a crucial role in clarifying these points to students.

- In the presentation of the uncertainty principle, we focus on sharply distinguishing the principle itself from the issue of errors or uncertainties resulting from measurement. We emphasize that the principle is about intrinsic uncertainties, which exist whether a measurement is performed or not (i.e., a limitation on the possibility to prepare a state with certain characteristics). Our initial introduction to the concept is based on the experiment of diffraction of photons or electrons from a slit of variable width. Later in the sequence, we connect the principle to the approximate determination of a minimum energy for bound systems.

- The sequence makes extensive use of simulations, designed using the open source software GeoGebra, both during classroom lessons and for exercises and home activities. The simulations we progressively realized to be incorporated in our TLS are described in our past published works $[7,40]$. Some of these concern cases that are traditionally considered in proposals based on the sum over paths approach, such as reflection and refraction of light, interference, and diffraction of photons and massive particles. Others model more advanced phenomena or experiments, which are less commonly considered, especially from a sum over paths perspective. In designing our simulations we tried as far as possible to avoid any interference with the classical paradigm, and, in particular, we never provided any representation of the quantum object itself; instead, the graphical interface shows the geometrical structure of the problem considered, the source and detector(s), the possible paths, and the sum of vector amplitudes producing detection probabilities.

- One of our guiding design principles is that conceptual understanding and the development of procedural abilities are inseparable, and that one can hardly advance without the other. In view of this, we took care of the problem of connecting the language of the sum over paths approach, which we used in our slides and lesson handouts, with the one used in the students' textbook, the Italian version of CutnellJohnson [41]. Our objective was that students could approach all the exercises in their textbook in the same way as they had done for all the previous topics of the physics course. In some cases, the work of adaptation included solving a textbook problem in class using a different method than the one suggested in the textbook. For example, the problem of a quantum particle-in-a-hole proposed in CutnellJohnson was approached with students using the fixed energy sum over paths approach described in Refs. [7,42,43]. Also, we assigned to students additional exercises specifically meant to have them familiarize with the sum over paths approach, which they were encouraged to solve collaboratively. Most of these exercises and problems are reported as Supplemental Material to this article [44].

- We devoted a substantial amount of time to discussing epistemological and metacognitive perspectives on the physical content. The reasons for this choice are (a) to encourage metacognitive reflection, which has been recognized as one of the factors favoring conceptual change [29], and (b) to achieve not only conceptual understanding, but also personal and emotional involvement of the students, i.e., to favor students' appropriation [45]. For example, at the beginning of the sequence we gave students a questionnaire, guided by quotes from physicists of the present and the past, on the different ways that a physical theory can be understood at the intuitive level (i.e., through images, analogies, or mathematical models). The questionnaire 


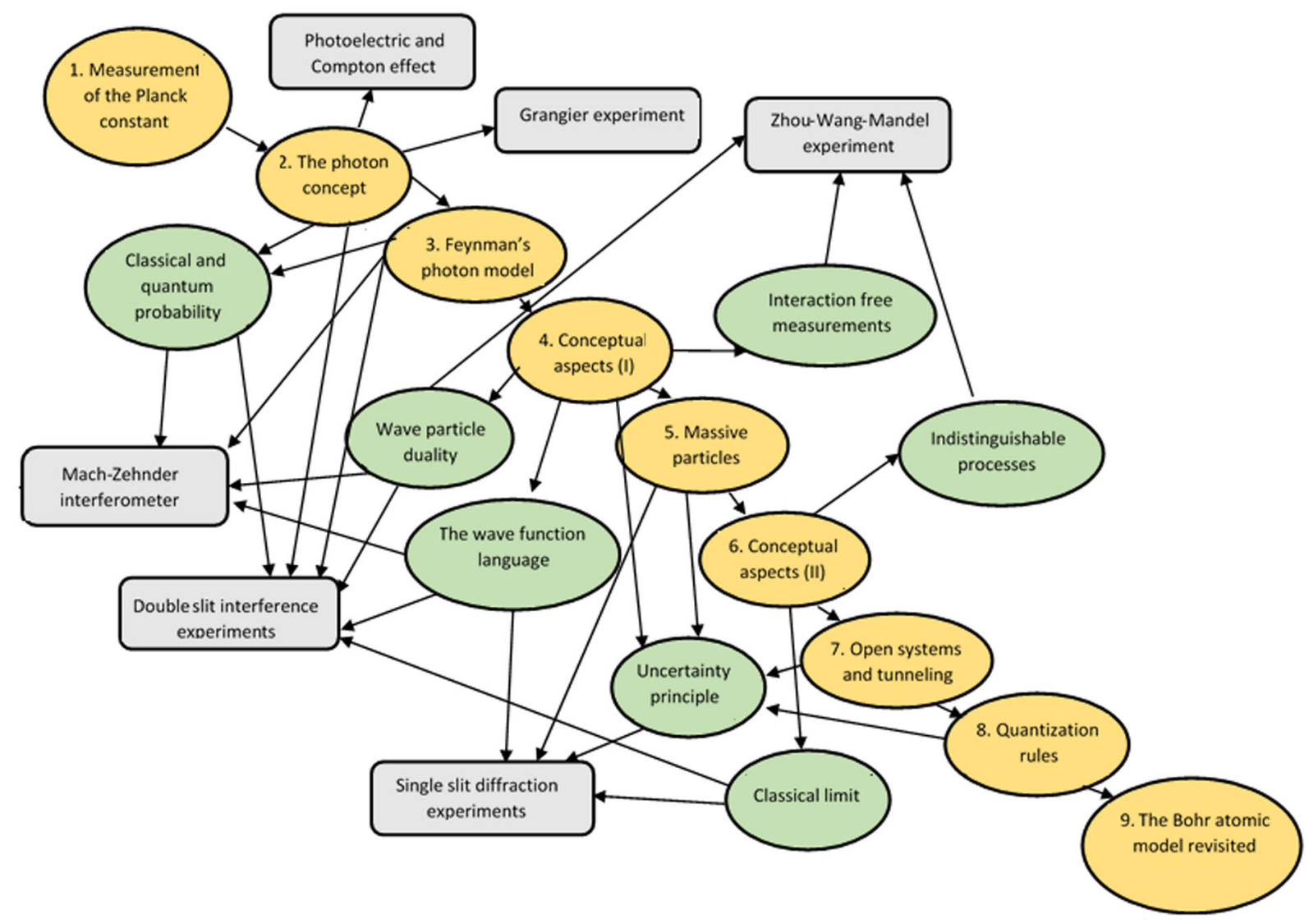

FIG. 1. The version of the TLS tested in high school. The central row (light orange) shows the main steps of the sequence, which is summarized in Appendix B. The green ovals are the most important conceptual themes and principles discussed, and the gray rectangles are the experimental evidence introduced.

was followed by an open classroom discussion in which students were encouraged to express their own ideas on the possibility of reaching an intuitive comprehension of the theory.

The full development of the sequence has been described in Ref. [28]. A schematic representation of the sequence is reported in Fig. 1, and more details on the content of each step are provided in Appendix B.

\section{Aims of this study (research questions)}

The general aim of this study is to evaluate the effectiveness of the sum over paths approach in the high school context. More specifically, a primary goal of our work is to test the hypothesis that the sum over paths approach could help providing students with a unified, integrated, and self-consistent picture of quantum theory. In order to reach these objectives, it is necessary not only to determine what mental models students build, and the incidence and nature of alternate conceptions, but also whether correct or partially correct models are connected in a consistent framework.

Although these two issues are largely intertwined, a separation line can be drawn based on the assessment tools which are used to study them. In previous literature on student's mental models of quantum concepts, the evaluation instruments largely consisted of either multiple choice questions or open response questions in which students were only asked to describe the meaning of a principle or concept, without being solicited to produce an organized reasoning with links to other ideas and phenomena. Investigating the interconnectedness of student's ideas requires different assessment tools.

In our study, KI assessment tasks (Sec. II B) and scoring rubrics (Sec. II C) were used to evaluate specifically the degree of integration and interconnectedness of students' acquired knowledge. Data obtained from KI rubrics were coupled to a more traditional attribution of student's answers to phenomenographic categories, to obtain multifaceted information on students' learning, including the degree of complexity and correctness of their explanations of individual phenomena, the incidence and nature of alternate models, and the overall consistency of their picture of quantum theory.

Based on the above premises, we divide the aims on this paper into two different, albeit closely related, research questions:

Q1: Can a teaching-learning sequence based on the sum over paths approach be effective in leading students to build 
scientifically valid mental models of the uncertainty principle and wave particle duality?

Q2: Can a teaching-learning sequence based on the sum over paths approach be effective in leading students to construct scientifically correct connections between different ideas, experiments, and problems related to quantum physics?

\section{METHODS}

\section{A. The classroom context}

The experimentation was performed in a class composed of 18 students, 10 male and 8 female, in the final year of secondary education, which in the Italian system is the 13th grade. The school is a "Liceo Scientifico," a type of secondary school oriented towards preparing students to a future university degree, primarily in the scientific field. However, the particular school where we tested the sequence is not an elite high school: it is positioned in a working class district; and its student population includes a significant percentage of first- and second-generation immigrants. We did not choose a particular class in the school for the experimentation; it was assigned by the school principal.

Indeed, the class in which the experimentation was performed had a known past history of poor results in mathematics and physics. At the end of the previous year, all students but four had been required to take a supplementary reparation exam in mathematics in order to progress to the next year classes.

The total amount of time devoted to quantum physics was about 33 hours, which is exactly the time suggested by the Italian guidelines for the physics curriculum of the final year of high school. The sequence was taught for 3 hours per week by their usual physics teacher, who is also one of the authors of this study (M.M.). The time for the sequence divided as follows: 3 hours for experimental activities; 9 hours for tests, questionnaires, and the production of argumentative papers; 2 hours reserved for open discussion; and the remaining 19 hours for traditional classroom lessons, including time employed for the sequence development, for the introduction of historical and epistemological themes, for providing the solution to exercises assigned as homework, and answering or discussing students' questions. In order to better envision how in practice the classroom and laboratory lessons were performed, we describe these settings in more detail:

- The classroom was equipped with a PC and an interactive multimedia board. We used slides on the multimedia board to focus on the main concepts of the lectures, and when necessary we switched to the internet (for examples, videos, or experiments in virtual labs) or GeoGebra (for the simulations). At the end of each lesson, the slides and relevant simulations were made accessible to students.
- The laboratory activities were performed in the context of an educational visit at the University of Pavia. Students were divided in small groups and given lab handouts following step by step the development of the activity. The photoelectric effect experiment was conducted using the PASCO $h / e$ apparatus. For the measurement of the Planck constant using LEDs of different colours [46] students had four LEDs (redyellow-green-blue), PASCO current-voltage sensors, and the Datastudio and MS Excel software. Students also used a homemade smartphone-based spectrometer [47] to observe various types of light spectra, such as a continuous, broadband one (sunlight) and a discrete one (the mercury gas lamp used for the photoelectric effect).

\section{B. Data collection and an overview of data sources}

In the course of the experimentation we collected data from a number of sources, including audio recordings of all lessons. Here we provide some more information on those data sources that are relevant to the present discussion:

Final test for school grading. - A final test for school assessment was performed by students about one week before the final conceptual questionnaire for research purposes. This test was designed to be similar to their usual classroom tests concerning other topics and to improve a perception of continuity between the research based TLS and their usual school experience. Only some of the exercises within the test for school grading are relevant to the present article, and will be discussed in Sec. III E 1

Final questionnaire for research purposes.-The main instrument to test the quality and integration of students' mental models of wave particle duality and the uncertainty principle was a conceptual questionnaire assigned to students at the end of the sequence. The questionnaire was not graded for school purposes, and students were informed of this fact. The questionnaire was composed of three KI assessment tasks, which were essentially openanswer questions requiring students to organize an argument about a complex problem. Two of the tasks (T2 and T3) concerned, broadly speaking, the wave particle duality aspect of quantum objects, the first focused more on photons and the Mach-Zehnder interferometer, and the second on electrons and the double slit experiment. The remaining task (T1) concerned the uncertainty principle, and required students to criticize (or agree with) an explanation of the uncertainty principle based on the accuracy-disturbance tradeoff and the thought experiment of Heisenberg's microscope contained in a common Italian high school textbook. The KI assessment tasks are reported in Appendix A.

Interviews to selected students.-Based on the lesson recordings and other data, six students were chosen to be individually interviewed. The criteria upon which the choice was based were the frequency of active participation in collective discussions, and the degree of personal 
TABLE I. Subtask division for tasks T1-T3.

\begin{tabular}{|c|c|c|}
\hline T1 (The uncertainty principle) & T2 (The Mach-Zehnder interferometer) & $\begin{array}{l}\text { T3 (Two slit interference with one } \\
\text { electron at a time) }\end{array}$ \\
\hline \multirow{2}{*}{$\begin{array}{l}\text { T1a: "Recognize that the presentation } \\
\text { is not consistent with the current } \\
\text { understanding of the principle and } \\
\text { explain" }\end{array}$} & $\begin{array}{l}\text { T2a: "Explain the outcome of the Mach-Zehnder } \\
\text { interferometer with one photon at a time (Fig. 7)" }\end{array}$ & $\begin{array}{c}\text { T3a: "Compare the result to the } \\
\text { expectation for classical particles } \\
\text { and explain" }\end{array}$ \\
\hline & $\begin{array}{l}\text { T2b: "Explain the outcome of the Mach-Zehnder } \\
\text { interferometer with one photon at a time and "which } \\
\text { way" measurement (Fig. 7)" } \\
\text { T2c: "Explain the apparent paradox and discuss the } \\
\text { properties of quantum objects highlighted by the } \\
\text { experiment" }\end{array}$ & $\begin{array}{l}\text { T3b: "Find connections and common } \\
\text { concepts with the case of the Mach- } \\
\text { Zehnder interferometer" }\end{array}$ \\
\hline
\end{tabular}

involvement in the subject of quantum physics, as highlighted by the content of their papers. Thus, the students interviewed were not necessarily the highest performing ones; and in fact one student who had performed rather poorly in the tests was interviewed. However, two students among the top performers were also selected based on the above criteria. All the students selected accepted being interviewed. The interviews were carried out by a researcher and co-author of this paper (P.O.) whom the students interviewed did not know before. The interview protocol was not of a clinical nature, but was more directed to understand students' personal involvement with quantum physics, and, in particular, the problem of whether they accept it from a philosophical perspective. This research goal was part of a different project [48] which involves researchers from other Italian universities, using different teaching approaches and strategies in other schools at the same level. In this case study, we present (in Sec. III E 2) excerpts from the interview to one especially successful student, with the aim of substantiating the claim that she has achieved a complex, integrated understanding of quantum physics. The analysis of passages of the interview, juxtaposed to extracts from her written productions, also shows in detail what kind of representation of quantum physics students can build through the sum over paths approach in a case of successful educational outcome.

\section{Data analysis}

In order to answer the two research questions, the answers to each item of the final questionnaire were analyzed according to two different methods:

- For each of the tasks, the principal model used by the student to account for the idea involved (wave particle duality or the uncertainty principle) was identified, using a phenomenographic approach. This allowed us to evaluate the appearance of alternate or hybrid models and perform a comparison with results reported by other authors.

- For the knowledge integration analysis, each task was divided into subtasks (Table I), roughly corresponding to the subquestions in the three principal questions (see Appendix A). Each subtask was then graded using a KI rubric (Table II) corresponding to the standard usage in the literature.

Subtask division is as follows:

Thus, T1 is a unique task, while T2 was divided into three subtasks, and T3 into two subtasks. This was made primarily to render the number of necessary scientific ideas to link in order to provide a complete argument for each subtask comparable; results analyzed in this way prove to be more informative than constructing a single rubric for each of the three principal tasks.

TABLE II. KI rubric used to grade answers to each subtask.

\begin{tabular}{|c|c|c|}
\hline Score & Level & Description \\
\hline 0 & No information & No answer to the given sub-task \\
\hline 1 & Irrelevant & Elicits ideas irrelevant to the context \\
\hline & & Elicits partially relevant ideas but answers a different question \\
\hline 2 & Non-normative or no link & Elicits non-normative ideas \\
\hline & & $\begin{array}{l}\text { Elicits relevant ideas but makes non-normative links between ideas } \\
\text { Links relevant and irrelevant ideas }\end{array}$ \\
\hline 3 & Partial link & Recognizes a link between relevant ideas but does not fully elaborate the link \\
\hline 4 & Full link & Elaborates a scientifically valid link between two relevant ideas \\
\hline 5 & Complex link & Elaborates two or more relevant links between relevant ideas \\
\hline
\end{tabular}


Note that there is clearly a significant overlap between the two methods of analyzing the answers, since answers in which a scientifically accepted model is used as a primary explanatory principle roughly correspond to grades of 4 or higher in the KI rubrics' scale [31]. However, phenomenographic analysis provides additional information on what alternate models students use in answers having a poor grade in the rubrics scale, while the KI rubrics emphasize information about the ability of students of connecting different ideas and phenomena in a consistent whole, so the two types of analysis complement each other.

The KI rubric used to evaluate each subtask is fairly standard and reported in Table II.

The relevant ideas to be elicited depend on the subtask. In particular, schematically a primary link between two relevant ideas can be identified for each subtask, which allows one to provide a convincing argument (Table III). Usually, when this link is fully elaborated the answer is assigned at least a 4 grade. In Table II we also report relevant "secondary" normative ideas, which may or may not be elicited by the student and connected to their argument to make it more convincing, or more complete. Answers in which such connections appeared with a link deemed scientifically valid, were assigned a grade of 5 . The distinction between primary and secondary ideas is to some extent artificial, but empirically it applies well to students' answers, in the sense that we did not find cases in which students could construct scientifically valid arguments bypassing the primary ideas entirely and using only the secondary ones.

TABLE III. Relevant ideas, divided into "primary" and "secondary," which have to be elicited and connected by students in order to provide a scientifically valid argument for each subtask.

\begin{tabular}{lc}
\hline \hline Sub task & Primary ideas \\
\hline T1a: "Recognize that the presentation & The principle is presented as the \\
is not consistent with the current & consequence of a disturbance due to \\
understanding of the principle and & measurement. \\
explain" & $\begin{array}{c}\text { Uncertainty is nowadays understood as } \\
\text { intrinsic indeterminacy. }\end{array}$
\end{tabular}

T2a:'Explain the outcome of the Mach-Zehnder interferometer with one photon at a time (Fig. 7)"

T2b:"Explain the outcome of the Mach-Zehnder interferometer with one photon at a time and "which way" measurement (Fig. 8)"

T2c:"Explain the apparent paradox and discuss the properties of quantum objects highlighted by the experiment"

T3a:"Compare the result to the expectation for classical particles and explain"

T3b: "Find connections and common concepts with the case of the Mach-Zehnder interferometer"
Quantum object follows all paths, each path has an associated vector amplitude.

Different paths giving the same experimental outcome interfere (quantum probability rule).

which way measurement makes paths of the quantum object distinguishable.

Distinguishable paths do not interfere (classical probability rule).

Contradiction with classical probability rule

Agreement with quantum probability rule (including the effect of which way measurement)

Classical corpuscle model predicts two

"spots" only, in correspondence of the slits.

Quantum objects follow all possible paths and produce interference.

Photons and electrons are both quantum objects.

All quantum objects are described by essentially the same mathematical model.
Relevant secondary idea(s)

Measurement does not necessarily imply physical interaction.

Uncertainty appears in contexts unrelated to measurement.

Single slit diffraction with variable slit width is a more faithful representation of the principle.

The amplitude vector associated to each path is a unitary vector with phase proportional to path length.

Beam splitters add a $\pi$ phase shift for internal reflection only.

In the new setup only one path per outcome remains, so probability is necessarily $50 \%$ at each detector.

The photon is an indivisible entity

The photon does not follow one single trajectory.

The photon is an indivisible entity.

The amplitude vector associated to each path is a unitary vector with phase proportional to path length.

Rotation rate in space is proportional to the inverse of de Broglie wavelength.

Bright spots correspond to points in which amplitude vectors associated to paths are in phase.

If in the two slit experiment a detector is placed at one of the slits, interference disappears, like in the Mach-Zehnder interferometer. 


\section{RESULTS AND DISCUSSION}

\section{A. Phenomenographic analysis: Results}

In the following tables we report the results of the phenomenographic analysis, where each category represents the main concept used by students in dealing with tasks T1-T3. $N=14$ students were present the day that the final test was scheduled.

\section{Wave particle duality}

In task T2 (Table IV) students had to explain the difference in the outcome of Mach-Zehnder interferometer experiment depending on whether "which way" information was available. $50 \%$ of students use a correct quantum model based on the photon following all possible paths, and the probability being computed using either the classical or the quantum rule, while 3 students only describe the outcome and provide a generic statement of duality. In one case, the answer is based on classical, deterministic ideas: the student interprets photons as classical corpuscles, and tries to explain the outcome by attributing selective properties to the beam splitters (they reflect photons with certain properties and transmit photons with other properties). One student seems to adopt a hybrid model consisting of the association of paths to trajectories followed with a certain probability.

In task T3 (Table V) students had to consider the result of a Young's experiment with one electron at a time, and to explain it in comparison to the classical expectation for both a corpuscle and a wave. Here, 9 students use a fully quantum model, while only 2 provide a generic statement of duality. One student provides an inconsistent answer based on a misunderstanding of the properties of classical waves, and basically offers no explanation for the quantum result. Two students provide no answer at all. We did not see any specific hybrid or alternate models appear in the answers to this task, although some may be hidden by

TABLE IV. Principal idea used by students in the answer to T2 (the photon and Mach-Zehnder interferometer).

\begin{tabular}{lc}
\hline \hline Model & $\begin{array}{c}\text { Frequency } \\
(n=14)\end{array}$ \\
\hline $\begin{array}{l}\text { Quantum model (quantum object follows all } \\
\text { possible paths, probability computed using }\end{array}$ & 7 \\
$\quad$ quantum rule) & 3 \\
$\begin{array}{l}\text { Vague duality (e.g., only phenomenologically } \\
\text { describes the experiment and provides a statement } \\
\text { of duality) }\end{array}$ & 1 \\
$\begin{array}{l}\text { Classical corpuscle, deterministic } \\
\text { Hybrid model (interprets paths as possible } \\
\text { trajectories, the photon only follows one path } \\
\quad \begin{array}{l}\text { although it is impossible to determine which one) } \\
\text { Incoherent or impossible to determine }\end{array}\end{array}$ \\
\begin{tabular}{l} 
No answer \\
\hline \hline
\end{tabular}
\end{tabular}

TABLE V. Principal idea used by students in the answer to T3 (the electron and two-slit experiment).

\begin{tabular}{lc}
\hline \hline Model & $\begin{array}{c}\text { Frequency } \\
(n=14)\end{array}$ \\
\hline $\begin{array}{l}\text { Quantum model (quantum object follows all } \\
\text { possible paths, probability computed using } \\
\text { quantum rule) }\end{array}$ & 9 \\
$\begin{array}{l}\text { Vague duality (e.g., only phenomenologically } \\
\text { compares the classical and quantum result and } \\
\text { provides a vague statement of duality) }\end{array}$ & 2 \\
$\begin{array}{l}\text { Incoherent or impossible to determine } \\
\text { No answer }\end{array}$ & 1 \\
\hline \hline
\end{tabular}

the fact that the task was probably easier than T2 (see Sec. III B 1).

\section{The uncertainty principle}

In task T1 (Table VI) students had to comment on a passage of an Italian textbook presenting Heisenberg's principle as due to unavoidable disturbance of the measurement apparatus on a measured system. Eight students adopted a full quantum model of intrinsic indeterminacy, while 2 agreed with the text presented. One student criticized the text citing Einstein's point of view that "God does not play dice" and expressed belief in a deterministic hidden variable model. In one case the model upon which the answer was based could not be decided, and 2 students did not answer the question.

\section{B. Phenomenographic analysis: Discussion}

In all three of the tasks, one-half or more of the students adopt in their arguments a full quantum model (8 out of 14 in T1, 7 in T2, 9 in T3). Most of these students display a precise and secure use of language, are confident in using terms in a personal way, and write rather long answers, with many cross-connections between one answer and another.

Some of the hybrid or alternate models reported in the literature appear in students' answers. Answers of this kind are usually shorter, sometimes contain disconnected sentences taken almost verbatim from the textbook or the sequence slides, and rarely try to connect one task with the

TABLE VI. Principal idea used by students in the answer to T1 (the uncertainty principle).

\begin{tabular}{lc}
\hline \hline Model & $\begin{array}{c}\text { Frequency } \\
(n=14)\end{array}$ \\
\hline Quantum model (intrinsic indeterminacy) & 8 \\
Measurement disturbs system (agrees with text) & 2 \\
Deterministic hidden variable & 1 \\
Undecidable, either quantum model or & 1 \\
$\quad$ unavoidable noise & 2 \\
No answer & 2 \\
\hline \hline
\end{tabular}


other. In the next sections we will discuss in more detail the results for the wave particle duality and uncertainty principle concepts, examining the alternate depictions emerging from our sample and performing a comparison with some results reported in the literature.

\section{Wave particle duality}

In tasks T2 and T3, 7 and 9 students, respectively, have been classified as adopting a full quantum model. This means that the students describe the difference between the behavior of classical and quantum objects in terms of the former, following all possible paths, and correctly stated the quantum probability rules as opposed to the classical ones. An example of one of the most concise (and not necessarily most exhaustive) of these answers to $\mathrm{T} 3$ is

"(...) because in quantum physics the effect of interference must be considered, which does not appear for classical particles. To compute the probability that an electron arrives at a given point, one cannot use the classical probability rule, but the amplitudes of paths must be considered: $P=|\psi(A)+\psi(B)|^{2}$. This formula represents the interaction of "possible" paths that the electron uses, provided these paths remain indistinguishable."

Three students in T2 and two in T3 only phenomenologically describe the outcome of the experiment or the difference with the classical expectation but give no further explanation, or the explanation does not appear to be connected to a consistent mental model. For example,

"A classical particle, in a two slit experiment, should behave like any liquid or granular material (water, sand...), so the fact that electrons produce bright and dark bands demonstrates that they have a wave nature. In this interference experiment one can find proof that electrons take all paths and have a double nature."

A small number of hybrid or alternate depictions of quantum objects appear in T2, but not in T3. One student in T2 depicts the photon as basically a classical corpuscle, although endowed with a wavelength. Interestingly, this student goes as far as writing down a completely made up theory of the behavior of beam splitters in order not to abandon his deterministic views; in fact he writes "the beam splitter has the property of transmitting photons only if they exceed a certain wavelength limit, otherwise it reflects them" and thus he goes on to explain how it is possible that in setup (a) all photons (of a certain wavelength) arrive to the same detector. In T3, the same student provides an incoherent answer. Another student rather clearly believes that in the Mach-Zehnder interferometer the photon only follows one path, although which one cannot be determined with precision; in fact she writes: "one cannot determine with certainty the path which the photon has taken and to which detector it will arrive"; "photons are indivisible and so they will take only one path". Although the same student appears to adopt a consistent quantum model in $\mathrm{T} 3$, it is possible that the same difficulty, which is a rather elusive one, is hidden in $\mathrm{T} 3$ by the fact that the task does not require considering the individual paths in detail.

In our study there does not seem to be any significant difference in students' mental models of the photon and the electron. Actually, in some cases students make revealing mistakes, as in the course of their writing they sometimes erroneously write "the photon" in place of "the electron" or vice versa, probably indicating a significant overlap between the two ideas in their conceptual framework. This idiosyncratic mistake could be investigated further, because it is possible that some students may have missed important differences between the two quantum objects, e.g., the fact that the electron is massive, which has consequences on how the wavelength is computed; but what clearly emerges from our data is that students are applying the basic mental model of quantum object to both the photon and the electron, which was one of the objectives of our teachinglearning sequence. This is in contrast with the largest study on high school students and wave particle duality available, the one of Olsen [17] mentioned in Sec. I, in which a very significant difference in students' mental models of the photon and the electron was observed following traditional instruction. In particular, in the cited study around 60\% of students attributed a mainly corpuscular ontology to electrons, but only $10 \%$ to photons.

Reference [17] is also a useful reference for a comparison with traditional instruction on the relative incidence of alternate models. In fact, in that study the answers classified as genuine duality were only $0.4 \%$ for photons and $2.5 \%$ for electrons, while those containing a vague formulation of duality were $59.2 \%$ for photons and $17.4 \%$ for electrons.

Our results on wave particle duality are scarcely comparable with those of the study by Müller and Wiesner [23] on German preuniversity students, because both the type of questions asked, the expected answers and the analysis methods are very different. However, one of the main general conclusions of such study is confirmed by our own results. They found that traditionally delivered preuniversity instruction in quantum physics is scarcely effective in leading students to build consistent mental models of quantum objects because it fails to offer a working functional model of wave particle duality, thus encouraging students to conceive vague dualistic depictions. Their approach, which obtains significantly better results than traditional instruction, is based on the Schrödinger wave function formulation. Their course in German high school is divided in two parts, as it offers a quantitative treatment of the subject (which requires basic notions of differential equations) only to a subset of students, who have a special interest in physics. Our study suggest that the sum over paths description or quantum objects constitutes an alternative, efficient way of providing student with a functional model of wave-particle duality, including the effect of 
"which way" measurements, using a much less advanced mathematical structure.

\section{The uncertainty principle}

In task T1, 8 students out of 14 criticized the passage proposed with arguments clearly based on the observation that uncertainty has to be understood as intrinsic property of quantum objects, in principle unrelated to measurement. For example, one student writes

"Heisenberg's idea is that it is our measurement to originate uncertainty on position and momentum, and that uncertainty arises when a photon is directed against the electron; actually uncertainty is properly intrinsic uncertainty always associated to the particle's position and momentum."

In one case, although the answer is in many respects similar to others which have been categorized as full quantum model, the choice of some words left in doubt on whether the student was actually thinking of intrinsic indeterminacy, rather than of intrinsic experimental noise; in fact, the student fails to realize that the uncertainty on one of the two complementary variables can be reduced at will, which is a common error reported in the literature for students holding this kind of alternate conception. Also, the student is the same one holding a clearly deterministic picture of the photon in T2.

Even though the task assignment rather explicitly suggested criticizing the presentation of the textbook, two students agreed instead with the passage on which the task was based and repeated or expanded Heisenberg's view of uncertainty as accuracy-disturbance tradeoff. One student, instead, criticized the passage proposed based on Einstein's view that "God does not play dice" and stated that uncertainty is presumably due to unobservable hidden variables that make the underlying reality deterministic.

Globally, with all the limitations previously discussed, our results seem at least not inferior to a trend line that sees, concerning the understanding of uncertainty as intrinsic indeterminacy, results going from less than one-third $[19,23]$ to about one-half (Ref. [22], which concerns student teachers) of the sample after instruction with traditional methods. However, the numbers of the comparison are certainly less impressive than in the case of wave particle duality. This may indicate that the sum over paths approach does not offer specific educational advantages in dealing with Heisenberg's principle; however, as we will discuss in the Sec. IV, there is also probably much room for further improvement in our treatment of the topic.

\section{Knowledge integration analysis: Results}

We summarize in Figs. 2-4 the grades obtained by students in their answers to tasks T1-T3, according to the KI rubrics displayed in Table II. In this case, we reserve all comments on the results to Sec. III D.

\section{Knowledge integration analysis: Discussion}

In the following sections we will discuss the results obtained by analyzing students' productions in response to the three tasks using the knowledge integration approach.

\section{T1: The uncertainty principle}

Task T1 can be concisely formulated as "Recognize that the presentation is not consistent with the current understanding of the principle and explain." The passage reported, which students had never read before as it was contained in a textbook other than their own, clearly presents uncertainty as a consequence of measurement by summarizing the Heisenberg microscope thought experiment; however, it does not explicitly formulate the idea that uncertainty is due to measurement. So, the "basic" connection that the student had to make in order to produce an argument for this task was to recognize the central idea contained in the proposed passage, and juxtapose it to the currently accepted interpretation. The 6 students who fully exploited this connection were attributed a grade of "full" or higher. Among the answers graded as "partial" are included two cases in which students correctly stated the accepted view of uncertainty as intrinsic indeterminacy, but made practically no reference to the proposed passage, probably indicating that they could not fully understand it.

The complete results for this task are reported in Fig. 2. As can be seen from the results, our attempts at connecting the uncertainty principle with different phenomena and aspects of quantum theory can be considered only a partial success. The three students who received a "complex" grade all elicited a different secondary idea and made a valid connection to it to reinforce their argument. In particular, one of the students recalled the idea that the uncertainty principle can be used to approximately predict the minimum energy of a bound quantum object. Another student reproduced the reasoning for obtaining the uncertainty principle from single silt diffraction with a variable

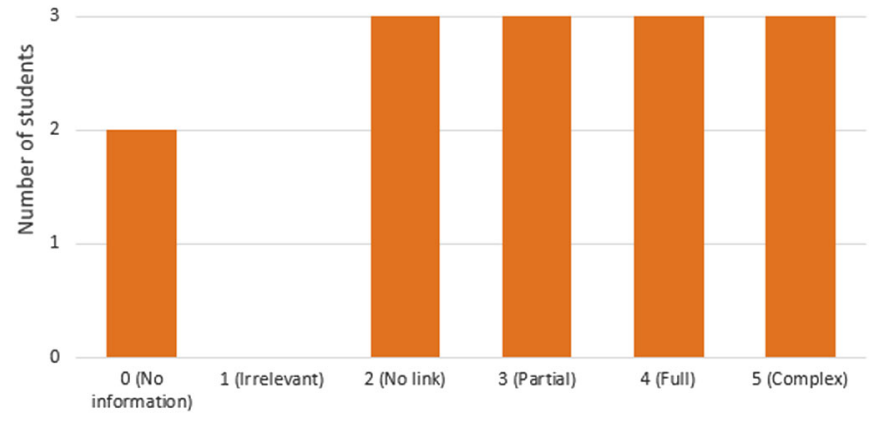

FIG. 2. Results for the task on the uncertainty principle (T1a "Recognize that the presentation is not consistent with the current understanding of the principle and explain"). 
slit width arguing that, in that case, no particle-particle interaction is involved. The third student evoked the ZhouWang-Mandel experiment as an example of the fact that a measurement can be performed without physically interacting with the measured object; such connection was not entirely expected, as the experiment is introduced in our sequence with the aim of clarifying aspects of wave particle duality related to which way measurements, but still the connection was considered as overall scientifically valid.

\section{T2: Wave-particle duality for the photon in the context of the Mach-Zehnder interferometer}

The knowledge integration analysis of task $\mathrm{T} 2$ and its subtasks (Fig. 3) represents essentially a measure of the quality of students' understanding of Feynman's model of the photon. In fact, in order to analyze the setups proposed (see Appendix A, Figs. 7 and 8), it is not sufficient to appeal to the basic concept that the photon follows all paths, but more specific ideas must be elicited. In task T2a, the four students who were assigned a complex grade all recalled that the beam splitter causes a $\pi$ phase shift for external reflection only, thus being able to completely explain the result. The two students who were assigned a full grade identified the possible paths of the photon, and explained how, in principle, probability of detection is computed. The idea that the phase associated to the quantum object is proportional to the path length was also elicited and connected to the answer by two of the students who received a complex grade, although this observation could be left implicit in the answer as the task assignment clearly stated that the two arms of the interferometers had the same length.

In a similar fashion, in task $\mathrm{T} 2 \mathrm{~b}$ the three students who were assigned a complex grade all connected to the idea that the presence of the which way detector makes the paths distinguishable, so that the classical probability rule applies; or equivalently that, for each outcome of the which way detector, only the paths corresponding to that outcome should be counted as "possible." At that point, the sum over paths algorithm is not even necessary to explain setup (b) (See Appendix A, Fig. 8) and indeed one of the students explained it appealing to photon indivisibility only.

In task T2c, "Explain the apparent paradox and discuss the properties of quantum objects highlighted by the experiment," students overall noted that the experiment is paradoxical in three distinct ways. It is paradoxical with respect to the classical probability rule, because it shows that the probabilities of events that are apparently alternative do not add up. It is paradoxical with respect to the classical corpuscle model, because it shows that photons do not follow one trajectory only; and it is paradoxical with respect to the wave model of light, because it shows that photons are indivisible entities since they are always revealed at one detector only. Answers were graded as complex if they highlighted at least two of these connections, and as full if they contained one of them, fully worked out.

\section{T3: Wave particle duality for the electron in the context of the two slit experiment}

The results for task T3 (Fig. 4) are highly significant for the purpose of answering the general research question about students' ability to build an integrated model of quantum physics. Subtask T3a required students to explain the contradiction between the classical corpuscle model of the electron and the results of the MerliMissiroli-Pozzi [49] two slit experiment with individual

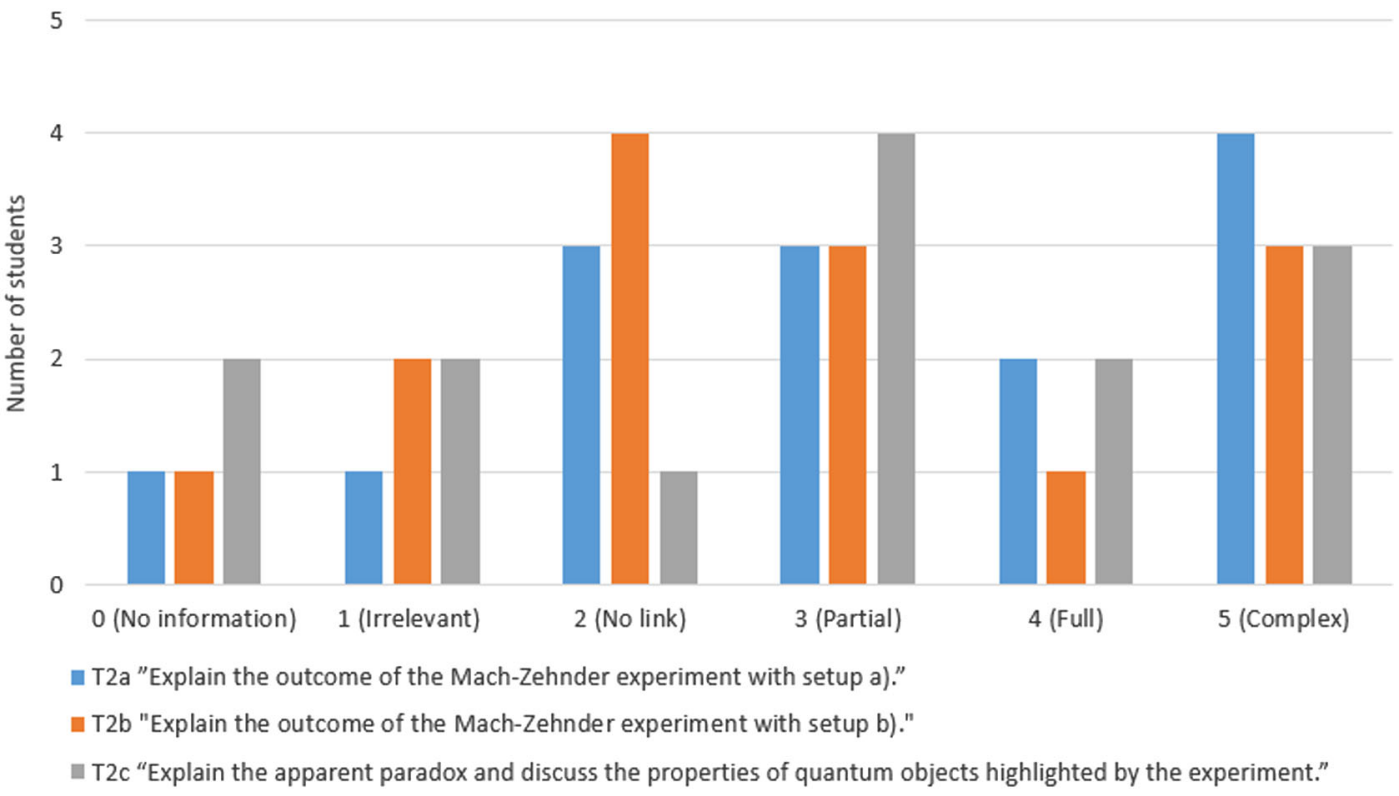

FIG. 3. Results for task T2 (wave particle duality in the context of the Mach-Zehnder experiment with one photon at a time). 


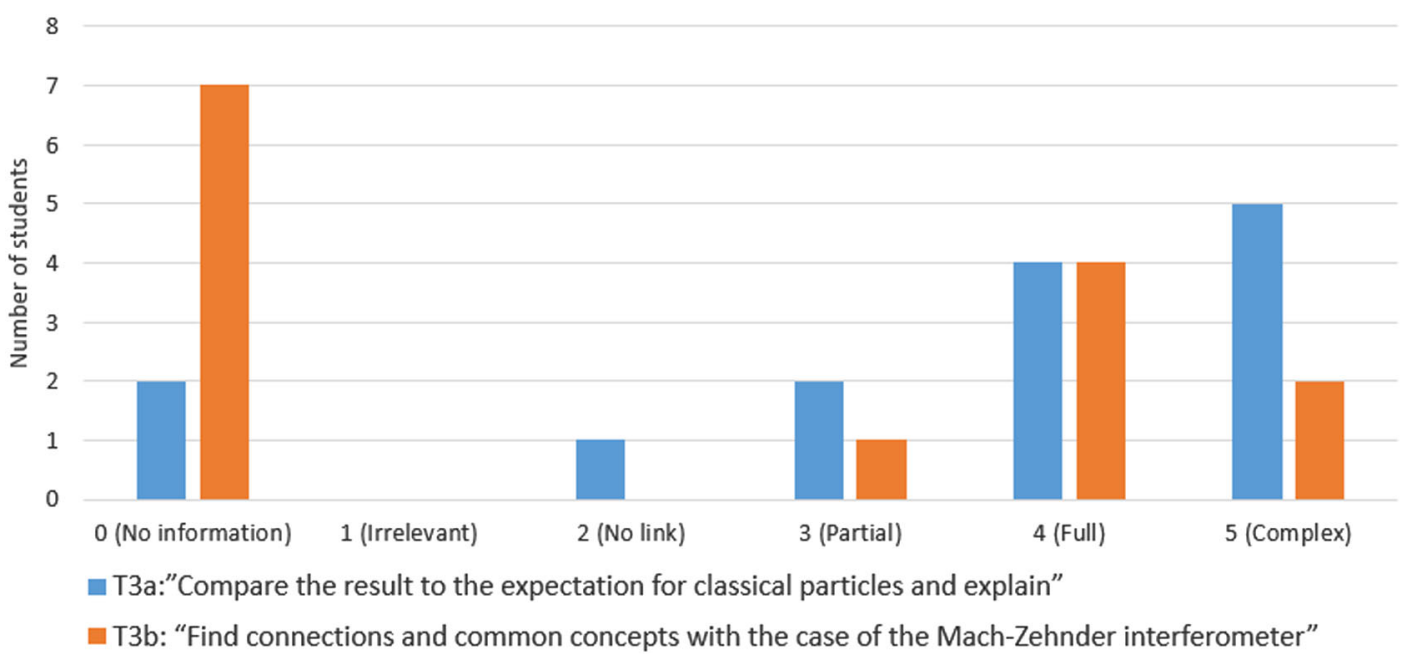

FIG. 4. Results for task T3 (wave particle duality in the context of Young's experiment with one electron at a time).

electrons. Results for this subtask were categorized as full if they contained a correct depiction of the expectation for classical corpuscles, and linked it to at least an in-principle statement of how interference is obtained if the quantum probability rule replaces the classical one. Answers graded as complex contained additional details; in particular one of the students worked out a full determination of the interference maxima, although this was not required by the assignment; while the other four students added the consideration that, in order to complete the calculation, the fact that the phase of the amplitude vector associated to a given path is proportional to its length must be considered. One of the students whose answers were graded as complex additionally included the formula to compute the de Broglie wavelength of the electron, while another remarked that the results of the experiment are in contrast with a purely wave model, since in that case uniform interference fringes would be predicted even at low intensity. The 9 students out of 14 who obtained a full or complex grade, demonstrated a good understanding of the sum over paths method in the context of the two slit experiment, which was probably more familiar to them than the MachZehnder interferometer.

Subtask T3b, in contrast, completely polarized the student sample. The subtask required highlighting connections and common concepts between the analysis of the Mach-Zehnder interferometer and the two slit interference experiment with one electron at a time. Half of the students did not provide an argument for this task at all, ${ }^{1}$ but the other half produced at least partially acceptable answers. The two students who were graded as partial all recognized one aspect of the analogy between the Mach-Zehnder

\footnotetext{
${ }^{1}$ Since this was the last item in the questionnaire, it is possible that in one or two of cases students did not answer this item because the time given was insufficient for them.
}

interferometer and the double slit experiment, in both cases with some imprecision or vagueness (for example, one wrote "The connection lies in the fact that both the photon and the electron follow all paths and have a double nature"). The other 5 all precisely highlighted that the behavior of both quantum objects is determined by the same model and the same quantum probability rule. In addition, the two students whose answer was graded as complex completed the analogy by observing that also in the case of the two slit experiment, if a which way detector is placed at one of the slits, interference is destroyed, like in the case of the Mach-Zehnder interferometer.

The result reinforces the conclusion, already drawn in the previous section, that students use basically the same model of duality for both the photon and the electron; in fact, it shows that half of them recognize explicitly, at least in an embryonic way, that the Mach-Zehnder interferometer with one photon at a time, and the two slit experiment with one electron at a time can be treated using essentially the same conceptual framework. Overall, our results on the tasks related to wave-particle duality reinforce the conclusion that the sum over paths approach is a viable option to pursue towards the objective of providing students with an integrated perspective on quantum physics in the context of an introductory high school course.

Looking at the other side of the coin, the fact that half the students do not provide an answer to task T3b, of course, also offers evidence of fragmentation of mental models: in fact, some of the students who did not answer the question had provided at least a partially valid analysis of either the Mach-Zehnder interferometer or the two slit experiment; in these cases, it is possible that the student is adopting an incomplete, only partially consistent model, constructed connecting memorized formulas or methods related to a given setup, without having in mind a selfconsistent whole. 


\section{E. Other data from the experimentation}

\section{Data from the test for school evaluation}

The final test for school evaluation was performed by students about a week before the questionnaire meant for research goals. $N=17$ students were present for the graded test (only one student was absent). The test was based on exercises similar to those found in their textbooks, not all of which are relevant for the present case study. In general, students did not show more difficulties than usual in solving textbooklike exercises, although instruction on quantum physics was based on a nonstandard approach. Compared to tests on different topics performed earlier in the same year, students results were better on average.

We hereby discuss briefly only two of the five exercises of the school test, which carry some relevance for answering our research questions. These exercises concern (1) wave-particle duality in the context of a two slit experiment for electrons and (2) a single photon Michelson interferometer. These two problems are reported in Appendix C. The results, as evaluated dichotomically as correct or incorrect are reported in Fig. 5.

In exercise (1), part (a) required computing the distance between two successive maxima for a two slit experiment with electrons, while part b) was a classical "what if" question that required imagining the outcome if a detector was placed at one of the slits. 9 students out of 17 correctly computed the answer to part (a), and 9 students also answered correctly part (b), providing a qualitative description of the loss of interference due to the insertion of a which way detector. Students predicted the formation of either a single large band of electron spots or two separate bands, one corresponding to each slit (both answers were judged as conceptually correct since the question did not require performing precise

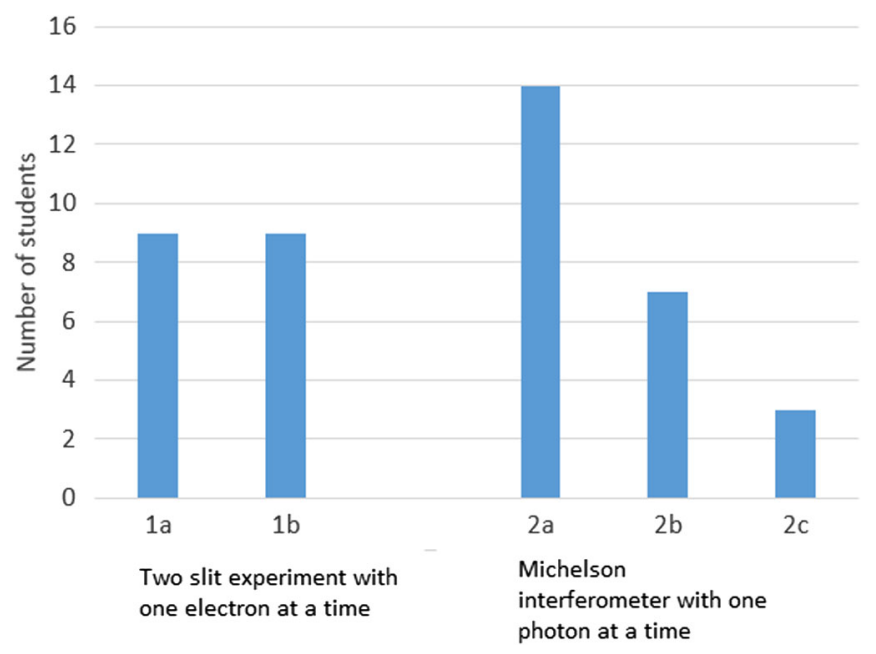

FIG. 5. Results (number of answers judged as basically correct) for each item in the final assessment test $(N=17)$. calculations). Seven students answered correctly both subquestions. These results agree well with the encouraging indications provided by the conceptual test concerning the understanding of wave-particle duality. However, it should be mentioned that three of the students whose answers were judged incorrect, while answering that interference would be lost, predicted that "nothing" would be seen on the screen. The above issue, along with other problematic elements appearing in the test, was discussed with students in the two lessons separating the assessment test from the conceptual questionnaire.

Exercise (2) was probably the most significant within the school assessment test for the purpose of contributing to our research questions, as it dealt with an experimental setup which students had never encountered, even by passage, during the sequence. The problem concerned the sum over paths approach for light in the context of a single-photon Michelson interferometer. The exercise required (a) enumerating the possible paths, (b) computing the detection probability, and (c) describing how to vary the setup in order to obtain the maximum detection probability. The ability of applying learned concepts, models, and methods to entirely new settings and phenomena is a key signature of successful knowledge integration [35]. The results are shown in Fig. 5: 14 students out of 17 could at least enumerate the possible paths of the photon which should be considered within the interferometer. Seven students were able to use the sum over paths method of adding up amplitude vectors to correctly compute the detection probability, with most incorrect answers (among the students who had successfully identified the paths) being due to neglecting the phase shift difference between internal and external reflection for the beam splitter. Three students only were also able to devise how the setup should have been modified in order to maximize the detection probability, a task that required a significant level of confidence in the sum over paths method. Not coincidentally, the three students providing a correct answer to exercise 3, part (c) would later all be among the top performers in the conceptual test. One of these students was Chiara, whose case we will consider in more detail in the following section.

\section{A more in-depth analysis of the case of Chiara}

In this section we will introduce a more detailed analysis of the conceptions of a single student, whom we will call Chiara. Chiara can be considered the clearest case of success of our intervention: she usually did not excel in physics, and her typical results were only average or slightly above average. This started to change during the development of the sequence. About halfway into it, Chiara was perceived by other students as one of the "leaders" in physics: they often appealed to her when they were not able to solve exercises, or when they wanted to discuss 
conceptual or mathematical aspects of the sum over paths method, of which she had acquired a very good understanding. At the end of the sequence, she did exceptionally well in both the graded and ungraded tests.

The analysis of the case of Chiara will be typically carried on by comparing side-by-side excerpts from her answers to the conceptual test, and passages from her final interview, accompanied by our own analysis of the indications we extracted from them. In some cases, issues which were not raised in the written tasks will be discussed, and only the interview excerpts will be considered. As discussed in Sec. II B, the interview protocol was not of a clinical nature, and its main objective was not to investigate conceptual understanding: the focus of the protocol was on personal and emotional involvement with the subject matter. However, the interviews did at times touch conceptual issues, and some of Chiara's utterances, when compared to her written statements, can shed additional light on the type and the robustness of mental models she has built. We next enumerate the most important elements of Chiara's discourse which could be gathered through our analysis:

(1) Like most successful students in this test, Chiara identifies the law for computing probabilities as a central element of difference between classical and quantum physics, and presumably a unifying, far reaching principle underlying quantum theory:

Interviewer: "So, according to you, what is the greatest difference between classical and quantum physics?"

Chiara: "Well I am thinking about probability, the probabilistic law."

I: "So in particular?"

C: "For example, in particular I was thinking that the probability of detecting a photon, in the case of interference, is equal to the square of the sum of amplitudes, while in classical physics probabilities are summed; and if a marble passes.. if marbles are thrown the outcome is a cusp after one slit and a cusp after the other one, and the probability is the sum of the two probabilities, but in quantum physics it is not so."

In the test (task T3a) she wrote

"If we modelled the electron as a classical particle, we would expect that, like a marble, it passes either through one slit or the other, and the interference pattern is not produced. The probability of detecting the electron would not be the quantum one $P=\left|\psi_{1}+\psi_{2}+\cdots\right|^{2}$ but the classical one $P=P_{1}+P_{2}$."

(2) Chiara is always very careful in distinguishing the level of reality from the level of the model in her discourse about quantum physics, and does not attach undue ontological implications to the elements of Feynman's model. This can be observed repeatedly both in the interview and the questionnaire. From the interview,

I: “(...) so how could you describe sum over paths, what is it?"

$\mathrm{C}$ : "In sum over paths to the quantum object is associated, in the mathematical model, a vector; and the vector makes one full turn for each wavelength..."

In another passage from the interview she explicitly remarks that the most important insights she got from classroom discussion were those about "how reality is described by a mathematical model."

Similarly, in her written answers to the questionnaire she carefully specifies that the quantum object following all paths is an element of the model, that vectors are associated formally to paths and so on. For example,

"To the quantum object is conventionally associated a vector, which makes a turn for each wavelength..."

(3) Chiara uses the sum over paths model to obtain a formal justification of the disappearance of interference phenomena due to a which way measurement, which is a key element of the concept of wave particle duality. From the interview,

I: "And how does the acquisition of information intervene in a quantum system?"

$\mathrm{C}$ : "Probably... if a system is detected, that is when information is acquired, the system should not be perturbed, but still it happens that interference disappears, because some paths are actually lost, so that for example if we have a certain probability of detecting a photon or an electron..."

I: "Ok I see you know it, but I wanted to know your opinion on the subject. That is does it surprise you that..."

C: "Well yes in fact at the beginning... in fact at the beginning I said well, maybe I misunderstood; but then the teacher said that indeed it seems like if the photon knows whether it is being detected or not, so it was rather paradoxical."

I: "And how did the teacher convince you?"

$\mathrm{C}$ : "Well, nothing, he convinced me."

I: "Yes but you were convinced because you trust the teacher or..."

$\mathrm{C}$ : "No, again for the same reason, because studying, making experiments, it became evident that things work this way. Also because of the mathematical model: if a path is removed, interference is destroyed, so..."

In the questionnaire (task T2b) she wrote

"In the second case the probability becomes $50 \%$ in $A$ and $50 \%$ in B because we inserted a detector which can reveal whether the photon passed or not through each of the two paths. Although the detector 
does not perturb the experiment, obtaining information on the paths taken changes the situation. If we know that a photon did not pass through a certain path, the probability changes because the possible paths are reduced in number."

(4) Chiara has a sense of "longitudinal" coherence of physics, that is she appears to be conscious that classical and quantum physics, taken together, should not provide an inconsistent picture of reality:

C: (...) let's say that in quantum physics particles have both wave-like and corpuscular properties, while in classical mechanics, this table, for example, does not have a wave-function characteristic."

I: "Eh, but then I am asking you: if I apply the de Broglie formula to the table..?"

C: "The formula for wavelength."

I: "Yes"

C: "Ok, it does have a wavelength, but it is so small that it is irrelevant. The biggest the object, the shortest the wavelength, so there is no interference.

(5) When discussing the uncertainty principle, Chiara repeatedly characterizes uncertainty as an intrinsic property of the quantum object, but also reports some difficulties in answering task T1 in a way she would have considered complete:

C: “(...) about the uncertainty principle, I understood well that it is an intrinsic principle for the quantum object, and that when one considers the relationship between energy and time, or position and momentum... and I understood the example, it was clear that if a quantum object passes through a slit, reducing the width of the slit the interference pattern on the screen becomes wider, which means that there is a greater uncertainty on momentum... but when in the test it was required to criticize a passage from a textbook, which clearly was not correct, I haven't completely understood which concepts were incorrect"

I: "Let's reflect about that."

C "I think in practice it was wrong because it said..."

I: "Ok apart from right or wrong, what do you think about uncertainty?"

C: "Eh, I think what I said, that it's a property of the quantum object, which evidently is not due to some error in our measurement."

I: "So it's just because the quantum object is... how do you want to call it?"

C: "Strange."

In her written task, she also insisted on the idea that the uncertainty principle is not due to some measurement error or disturbance:

"Quantum objects have as intrinsic some properties which may seem paradoxical, such as uncertainty concerning position and momentum."
"Indeterminacy is an intrinsic quality of the quantum object, and is not due to inaccuracy in measurement."

As Chiara reports in the above interview excerpt, in her answer to the test she offered the example of single slit diffraction with a variable slit width as a better representation of uncertainty than the one proposed by the task statement. It is possible that her perceived difficulty could be in distinguishing whether the presentation reported was actually wrong, or rather not fully adequate, because it could not account for all the possible instances in which quantum uncertainty plays a role.

(6) When asked to provide a compact representation of the core content of quantum physics, Chiara brings forward the two slit experiment. Especially in the final part of the passage the description is impressive because, rather than repeating expressions heard from the teacher or read from the textbook, Chiara seems to be finding her own, quite appropriate words for describing the experimental result.

I:" At the beginning of the sequence you discussed of the possibility of representing physical concepts with images, formulas or analogies. If you had to do the same with quantum physics.."

$\mathrm{C}$ : "I think pictures."

I: "You would use pictures and what would you draw?"

C: "The experiments with slits."

I: "Ok, but remember this is not a school test, you have to say what really comes to your mind when it comes to represent quantum physics intuitively. For example another student told me about photons like marbles in the photoelectric effect..."

C: "No, I would think instead of an experiment in which one sees in parallel the interference of a wave, and the case of marbles, and then the fact that in quantum physics they are superposed that is we do not see like the marbles but we see interference.."

I: "So you thought of parallel images, that is..."

C: "Yes, I imagined what we see with particles, what we see with a wave, and what we see with the quantum object, which is wave, but if you detect it, it's particle."

(7) Finally, in the course of the interview, Chiara shows sign of metatheoretical reflection on the logical structure of quantum theory, and metacognitive awareness of where her common sense intuition may fail in the new context. Such signs are scattered in several parts of the transcript, but can be effectively summarized by the following quote, in which Chiara explains that studying quantum physics led her to reflect on the relationship between physical models and logic, and between logic and ordinary common sense: 
I: "Did quantum physics satisfy your requirements for logical rigor?"

C: "(...) Well it depends, my idea was about classical logic, but it depends on how you interpret the concept of "logic". Originally, I thought that logic was simply common sense, what we are used to think. Then I reflected upon it, and I said to myself: "Logic is not simply common sense." So I asked to myself: "What is logic?" and I started from Aristotle, deduction rules... then I learned about mathematical logic..."

I: "But according to you, what principles of classical formal logic are contradicted by quantum physics?'

C: "Certainly the law of excluded middle, the idea that if $A$ and not $A$ are given, they are incompatible. Instead it seems that the quantum object, for example, is not just either here or there..."

We may now draw some conclusions for the case of Chiara: not only does she show a solid conceptual understanding of the new paradigm of quantum physics, and appeals to far-reaching principles to provide a unifying description of the theory (points 1, 3, and 6), but she also displays elements of discourse that several have associated with conceptual change [29,50,51], such as epistemological reflection and restructuring (points 2 and 4), metacognitive and metatheoretical awareness (point 7). It's worth specifying that we are not hereby stating that Chiara has achieved "expert" knowledge about quantum physics, but rather that she has successfully set the founding stones of its conceptual paradigm, upon which, maybe, she can build later.

Of course not all the interviews provided as many positive indications as in the case of Chiara. For the poor performing student interviewed, whom we will call Luca, the interview allowed us to more precisely characterize his mental models than the test had done. Luca had attached an overconcrete interpretation to the sum over paths model, and saw the rotating arrow as the representation of an actual rotation of the particle. See, for example, the following two quotes:

L.: "No, I think [the rotating arrow] it's a mathematical tool but I don't know if it corresponds... does it correspond to an actual rotation of the particle?"

L: "Concerning classical probability, if two events are mutually exclusive then the probability is equal to the sum of the probability of $A$ and the probability of $B$, while in quantum physics it is not so because this rotation movement of the particle is acting... right?"

This issue was first reported as a possible undesirable educational outcome by Jon Ogborn in 2006 [52] in the context of a qualitative evaluation of five years of teaching quantum physics in high school by the sum over paths approach. In our sequence, we put a significant emphasis on the idea of the sum over paths algorithm as an abstract mathematical model not to be put in one-to-one correspondence with reality. However, considering both the positive impact that this idea seems to have had in organizing Chiara's mental representations and, on the other hand, the negative consequences that may result from neglecting such warning, such as in the case of Luca, it is possible that our sequence may profit from stressing the point even more.

\section{F. Validity and reliability issues}

\section{Validity issues}

The main factor limiting this study is, of course, the sample size. $N=14$ students who participated to the final conceptual test do not offer a statistically significant enough sample to draw definitive conclusions. It may be, however, worthwhile to remind again that the starting level of the sample in physics and mathematics, with respect to other students of comparable age and school side, was considered to be on the lower side of the average, so that at least it can be excluded that good results are due to experimenting in a class especially interested or high performing in physics.

In our study we did not include any form of pretest to assess initial knowledge, assuming that in the case of high school students with no previous formal training on the subject, preinstruction conceptual understanding of quantum physics should be considered virtually nonexistent. Such assumption is consistent with our direct observation of the classroom, as we had no signals of pre-existing personal interest on the topic from any of the students. However, the possibility still exists that some of them had acquired notions of modern physics from the internet, TV documentaries, or other sources. Although we judge unlikely that any structured picture of quantum theory was built in this way, this limitation should be considered when evaluating the validity of our study.

Another issue which must be considered in assessing the validity of the present study is that, although the quantum physics sequence was taught by the regular school teacher of the classroom, this teacher was also one of the designers of the sequence. Thus, the addressing of transfer issues (dissemination of the proposal, formation of teachers to the task of implementing it in their own classrooms, and new tests of the results) must be considered as a first priority for future work.

\section{Reliability issues}

The present being a small study, with few participants and questions, it is not possible to conduct analyses of intrarater reliability and internal consistency of the questionnaire; we focus here on the issue of interrater reliability

Concerning the phenomenographic analysis, the categorization of answers was conducted collectively 
by three of us. Decisions about placing answers in one or the other category were taken by agreement of the evaluators. In a few cases, decisions were taken by majority. The percentage of unanimous decisions was about $90 \%-95 \%$.

For the KI analysis, the rubrics were created and the answers were rated independently by each author. The final scores were attributed by the majority. For the KI rubric scores assigned by the three raters, the Cronbach alpha was 0.96 , the percentage of total agreement between the three raters was $60 \%$, and the percentage of adjacent agreement was $93 \%$.

\section{CONCLUSIONS}

According to our results, the sum over paths approach may be effective in overcoming some of the educational difficulties in the teaching of basic concepts of quantum physics. Feynman's approach offers a natural functional model of wave particle duality, which helps students build consistent, detailed, and integrated mental models. A majority of high school students are able to construct at least a partially integrated view (score of 3 or more in our KI rubric) of different quantum phenomena and experiments concerning wave particle duality and which way measurements, and 9 students out of 14 use the sum over paths approach to provide a fully consistent explanation of the two slit experiment with one electron at a time. In addition, our data do not exhibit the discrepancy between students' models of the photon and the electron which had been highlighted by several, and one-half of the students in our sample explicitly recognize at least some aspects of the parallel between the two mental models. The incidence of deterministic and hybrid conceptions of wave particle duality is limited in our data, and in particular the difficulty reported in the literature, consisting in believing that possible paths correspond to trajectories followed by the quantum object with a certain probability, was limited to one case only in our sample, presumably thanks to proper sequence planning. We emphasize that the result of this study concerning students' construction of satisfactory models of wave particle duality is not isolated, but is consistent with two previous studies, admittedly both of which are also small, reporting the same conclusions, with much higher percentages, in the case of teacher training $[7,28,40]$.

Concerning the teaching of the uncertainty principle, the interpretation of our data seems less clear cut. Again, results are consistent with those previously obtained in the context of teacher training [7]. A majority of high school students identifies the uncertainty principle as an intrinsic property of quantum objects, and not as a consequence of disturbance or a measurement error; but only three students are able to connect in a coherent way the principle to different phenomena and experiments in which its significance may be appreciated. Our results on students' mental models seem only marginally better than those obtained using the traditional approach, and among the research-based proposals, the one by Müller and Wiesner [23] achieves better results than our own, although probably in a more favorable educational setting. It must be noted that the cited authors also adopt a strategy involving the presentation of the principle from different perspectives: in fact, they use both a statistical approach (the uncertainty principle as statistical distribution of measurement outcomes on identically prepared systems) and one which focuses on indeterminacy on complementary variables for an individual quantum object (based on single slit diffraction with variable slit width). We believe that the combination of these results supports the conclusion that approaching the principle from different points of view, including epistemological and experimental ones, and connected to problems in which it has a specific explanatory value, is effective in supporting students' conceptual understanding. However, the connections between these points of view should also be strengthened to provide students with a coherent, integrated view.

Several recent studies have highlighted that students' mental models of quantum physics after introductory courses are fragmented, and consistent only in the context of an individual experiment or phenomenon. In view of these results, it appears urgent to focus research not only on the correctness of students' mental models of individual concepts, but also on their interconnection and organization into an integrated framework. This study constitutes a first step in that direction, conducted using the tools of knowledge integration theory.

\section{ACKNOWLEDGMENTS}

The work was funded in part by the Italian project PLS Piano Lauree Scientifiche - Progetto Nazionale di Fisica, Italian project code PN157YP17B. We acknowledge that assessment tasks of the final questionnaire (Appendix A) were developed in collaboration with Professor Olivia Levrini and Dr. Giulia Tasquier of the University of Bologna. We wish to thank the Principal of Liceo Enrico Fermi, Dr. Michele Lattarulo, for his support and collaboration during the experimentation.

\section{APPENDIX A: QUESTIONNAIRE USED FOR DATA COLLECTION}

Task T1-Quantum uncertainty [the question text was accompanied by Fig. 6]

In the textbook by U. Amaldi (in the 2003 edition), the uncertainty principle is presented in the following way:

"In order to "see" a particle, we must have it scatter the light directed towards it in such a way that part of the scattered light arrives to our eyes, or to detection instruments. To do so, it is necessary that the wavelength of light is at most equal to the length scale of the item which we desire to "see" (...) but the photons which form a light ray 


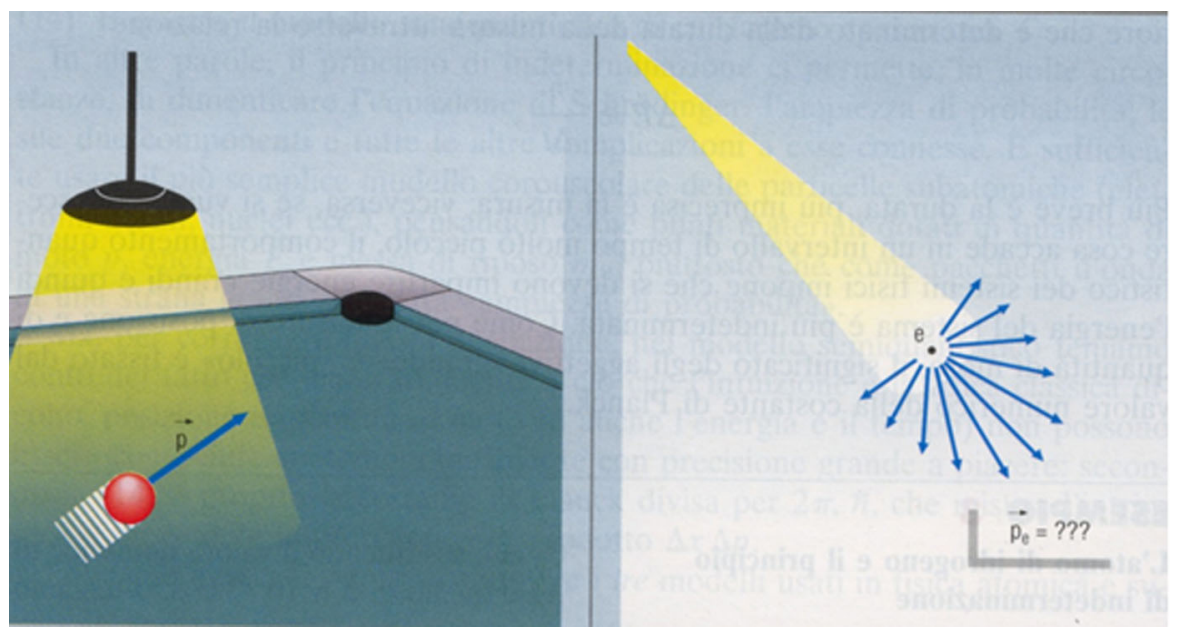

FIG. 6. Depiction of the uncertainty principle from the Heisenberg microscope perspective (reproduced from Ref. [53]).

with small wavelength (...) are very energetic and interact with material particles producing Compton scattering."

"In conclusion, the particle which we can see, because it has been hit by a photon which has then arrived to our detector, has undergone a collision which accelerated it in a random way. Thus, after the measurement, we can know its position, but we lost all possibilities of precisely determining its momentum. It is interesting to note that if we wish to have a lower uncertainty $\Delta x$ on the position, using light with a lower wavelength, the energy of incident photons must be increased, and as a consequence, the uncertainty on the particle's momentum increases."

Comment on this presentation, answering to the following questions:

(i) What criticisms can be raised to this introduction to uncertainty?

(ii) Which examples can be made to show that the discussion in the textbook is not adequate?

Task T2-The Mach-Zehnder interferometer

In Figs. 7 and 8 the results of two possible experiments using a Mach-Zehnder interferometer are shown. The first case is the ordinary one, and the probability of detecting the photon is $100 \%$ at detector B. In the second case, using an

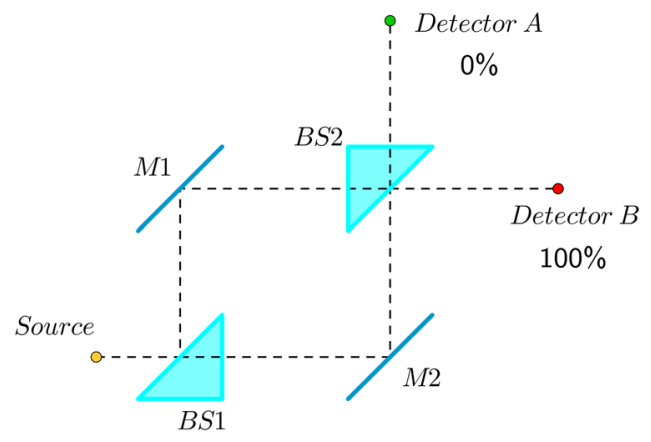

FIG. 7. The ordinary Mach-Zehnder experiment and its results (the two arms of the apparatus have the same length). intermediate detector $\mathrm{C}$, which detects the passage of the photon (without destroying it, and ideally without interacting with it) the resulting probability is $50 \%$ for each one of the two detectors.

(i) Describe and analyze (briefly, but also in a formal way) the experimental apparatuses in Figs. 7 and 8 and explain the experimental outcomes.

(ii) Which properties of the quantum objects are highlighted in these experiments? How would you convince someone who does not know about quantum physics that such aspects are surprising but not incomprehensible?

Task T3-Two slit experiment with one electron at a time

Referring to Fig. 9, which reports photographs of the Merli-Missiroli-Pozzi experiment of two slit interference with one electron at a time after a progressive number of electrons has deposited on the final screen, discuss the following points:

(i) If we modeled the electron as a classical particle, the result seems to be in contradiction with the principles of probability. Why? How can the paradox be resolved through Feynman's model?

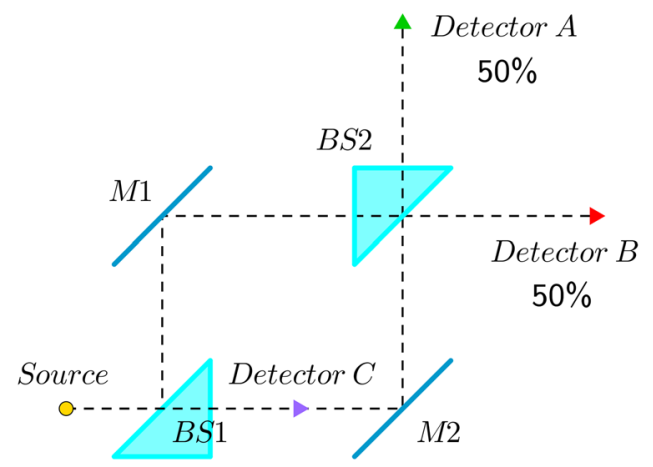

FIG. 8. Results for the Mach-Zehnder experiment with an intermediate detector $\mathrm{C}$, which can reveal the passage of the photon without destroying or perturbing it. 

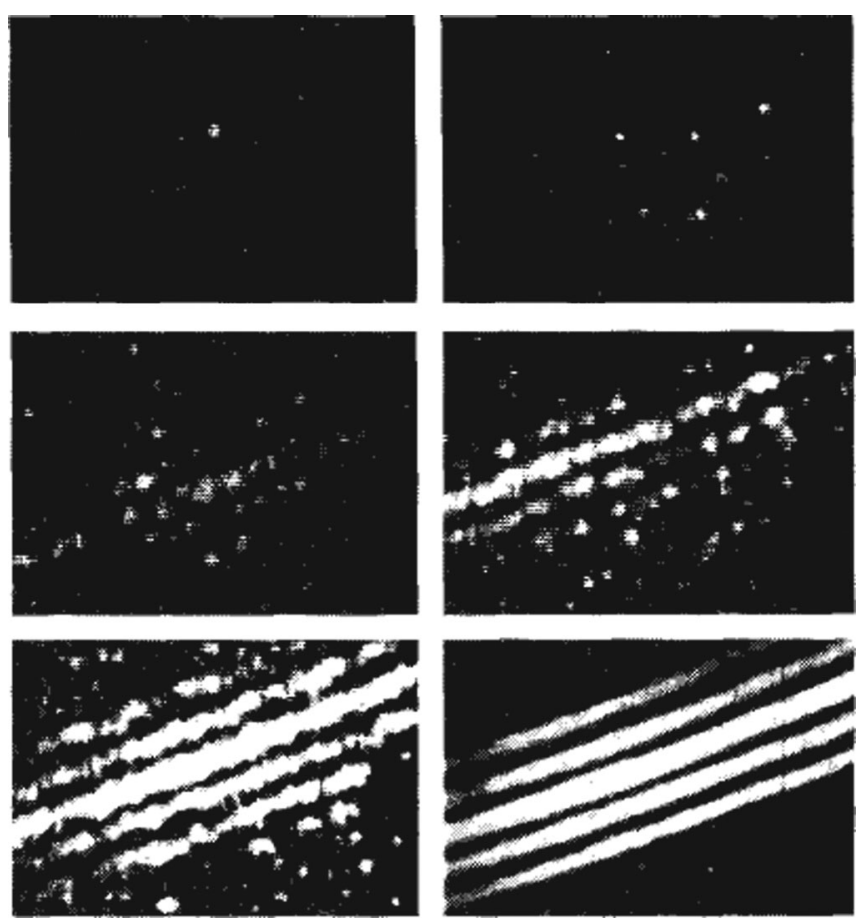

FIG. 9. The Merli-Missiroli-Pozzi experiment 1974-76, reproduced from Ref. [49] with the permission of the American Association of Physics Teachers.

(ii) What conceptual instruments can be taken from the analysis of the Mach-Zehnder experiment in item 2 to interpret the Merli-Missiroli-Pozzi experiment also?

\section{APPENDIX B: SCHEMATIC DESCRIPTION OF THE CONTENT OF THE SEQUENCE STEPS}

In this Appendix we describe in some more detail the disciplinary content of the sequence steps (Fig. 1). All the content described is treated at the level of mathematics complexity appropriate for the 13th grade of Liceo Scientifico. Complex numbers are not used (rotating arrows, which can be interpreted as phasors, are used to represent them) and, although students at this level are expected to know basic calculus, this is not used in the sequence, except maybe for some textbook exercise.

\section{A. Measurement of the Planck constant}

Students started the sequence in the physics laboratory, measuring the Planck constant with two different methods, well known in the literature: by using the photoelectric effect apparatus, and by measuring the activation voltage of LEDs of different colors [46]. Thus, the contradictions with the wave model of light and the necessity of a photon model are first demonstrated in the context of experimental activities.

\section{B. The photon concept}

We describe this point in more detail, as it constitutes a significant example of how our design strategy attempted to prevent students' formation of hybrid models of quantum objects.

Students have to be first given concrete evidence that the photon exists: discussing traditional evidence (i.e., the photoelectric and Compton effects) may be efficient in this respect. The existence of the photon, however, implies that the overwhelming evidence in favor of a wave model of light should be discussed in terms of photons. For example, if the photon exists, then how is the interference pattern in Young's experiment formed? It is known in the literature that, if the point is not addressed, students may form a hybrid conception of the pattern appearing as the result of interactions, or interference, between one photon and another. Thus we show students a video [54] of the accumulation of the two slits interference pattern using a very weak light source that emits one photon at a time.

The conclusion to which we would like to lead students is of course that the photon interferes with itself; however, students may try to save their classical world vision by hypothesizing that the photon actually splits in two at the slits and then recombines. Thus, at this point, we discuss the Grangier experiment on the indivisibility of the photon.

Only after having presented decisive evidence to counter the possible appearance of mixed classical quantum conceptions, we introduce Feynman's model of the photon following all possible paths from the source to the detector, arguing that, notwithstanding its prima facie absurdity, it can explain all the previously considered evidence in a consistent way.

Still, students may perceive the model as only a sort of metaphoric representation of physical reality, and believe that, at the end of the day, photons only follow one of the possible paths that the model assumes them to explore. This is a possible specific difficulty connected to the use of Feynman's approach which has been hypothesized by some [55]. Our approach to counter this problem is based on a detailed analysis, made also with the help of a computer simulation, of the results of a Mach-Zehnder experiment with one photon at a time, and the comparison of the full setup with the one in which either one of the two arms of the apparatus is removed or blocked. The resulting detection probabilities in the different cases very sharply exclude the hypothesis that, in the full setup, the photon has taken only one or the other of the two possible paths. The result is then extended, beyond the particular setup, by considering the analogous case of the two slits experiment, and comparing the resulting probability distribution with the one corresponding to the cases that either one of the slits is blocked. 


\section{Feynman's photon model}

In this step we introduce the basic sum over paths algorithm for a typical source-to-detector problem involving a single photon with defined energy, basically in the same way as done by Feynman in the first part of QED. Using such formalism, we discuss various problems, such as interference, diffraction, refraction, and so on, with the help of GeoGebra simulations.

\section{Conceptual and foundational aspects (I)}

This part of the sequence comprises three main elements: (a) The single photon Mach-Zehnder interferometer as a demonstration of the impossibility to attribute a single path to a quantum particle.

(b) Diffraction of a photon from a single slit with a variable width, introducing the uncertainty principle [56].

(c) The Zhou-Wang-Mandel [39] experiment used to highlight the role of which way information.

Discussion and/or simulation of the Mach-Zehnder interferometer has been used by others in education [23]. The primary function of the Mach-Zehnder interferometer in our sequence is to provide a convincing proof of the untenability of the classical trajectory concept. Comparing, also with the help of a simulation, the outcomes corresponding to different setups of the apparatus proves that the photon cannot be thought as taking either one or the other of two possible ways, but must be imagined as taking both of them simultaneously. In other words, it is impossible to associate a single path to a quantum object. Exercises on the Mach-Zehnder setup are also very useful for introducing to students the concept of normalization of wave functions, and for improving their understanding of the quantum rule for probabilities.

The Zhou-Wang-Mandel apparatus is a two way, single photon interference setup where which way information is collected without any physical interaction with the photon arriving at the detector where interference is revealed, through a clever use of nonlinear crystals. The setup is essentially analogous to a two slit experiment with the possibility of placing or removing an ideal detector, which detects the passage of the quantum object at one of the slits, ideally without perturbing it [57]. The discussion of the Zhou-Wang-Mandel experiment plays a crucial role in our sequence: we complete the picture of wave particle duality by treating the consequences of which way measurements, and clarifying the difference between indistinguishable processes (for which the quantum probability rule is used) and distinguishable ones (which obey the classical probability rule).

In this part of the sequence we also briefly discuss the connection between Feynman's approach and the wave function language; in essence, the wave function is nothing else than the complete set of "amplitude arrows" associated to a quantum object at a given point in space and time.

\section{E. Massive particles}

In this section we first discuss with students and show them evidence demonstrating interference effects for electrons [49], neutrons [58], and even C60 molecules [59]. The Feynman model for the electron is constructed by analogy with the one valid for the photon, by rewriting the expression for the phase of the vector amplitude $\varphi=$ $k x-\omega t$ in a form valid for massive particles. Thus, the expression for the de Broglie wavelength is derived. However, it is important to highlight with students that, although the conceptual models of massive and massless quantum objects are effectively identical, some formulas should not be confused; for example, one should not try to apply $E=h \nu$ to the electron, a common student difficulty reported by some [60].

\section{F. Conceptual and foundational aspects (II)}

In the second of the steps of the sequence devoted to the discussion of more abstract conceptual and foundational aspects, we discuss basically two issues: (1) the in-principle generalization of the sum over paths approach to more complicated problems involving processes with more than one particle, and (2) the correspondence principle and the classical limit.

Concerning the first issue, we reconsider the ZhouWang-Mandel experiment reinterpreting the result as showing that interference is not a phenomenon connected to one or the other photon, but to the possible alternative, indistinguishable processes leading to the same experimental outcome. In the sequence tested in high school this was little more than a brief hint, but in future realization we plan to expand on this point, as we already have done with student teachers $[40,61]$ by introducing the formal analysis of the Hong-Ou-Mandel experiment.

The classical limit is introduced with the essential help of simulations in which the wavelength (or the mass) of the quantum object can be interactively varied. When the wavelength of the quantum object becomes much smaller than the relevant length scales, the sum over paths approach reproduces the results of a classical theory. For the photon, the dominant path in this limit is the one predicted by the Fermat principle, thus retrieving the ray of geometrical optics. The behavior of massive particles, on the other hand, approaches the one predicted by the condition of stationarity of the reduced action, leading to the correspondence principle and explaining the emergence of classical mechanics as an approximate theory. Examples shown to students include light refraction at an interface, parabolic mirror reflection, and diffraction of massive particles at a double slit.

\section{G. Open systems and tunneling}

Towards the final part of the sequence, we introduce a semi-open, resonant system, in which the detection 
probability depends very strongly on energy, showing sharp resonance features. Such discussion is intended to provide a smooth learning path from source-to-detector problems to bound systems. The system, in the optical case, consists of a source emitting monochromatic photons towards a detector, with two successive beam splitters interposed along the path. In the case of a massive particle the role of beam splitters can be played by thin potential barriers of variable height, especially tuned in such a way that for any given energy the square modulus of the transmission coefficient is always $|t|^{2}=0.5$. By varying the energy $E$ of the incoming quantum object, one observes that the detection amplitude goes from a very low minimum to very sharp maxima, which, as usual, corresponds to the case in which all amplitudes associated to two paths differing of a full back and forth reflection in the two barrier system are in phase. This system does not yet have a discrete set of energy levels; however, it strongly selects some values of energy, for which the probability of detection is much higher. The same basic conceptual tools allow us to discuss, also with the help of a simulation, the problem of tunneling from a square barrier.

\section{H. Quantization rules}

In this section we introduce the concept of quantization of energy for a bound quantum system. First, we deal with the problem of the particle in an infinite square well, finding the allowed energy levels through the fixed-energy sum over paths approach $[42,43]$ and discussing the stationary wave function with the help of a simulation. The exact result for the ground state energy of the infinite square potential well is then compared to the approximate one which can be found using the uncertainty principle, and the generality of the result is highlighted. Then, we turn to the problem of a quantum object confined to a circumference, which we deal with using the same approach, i.e., we consider the sum of all paths at fixed energy going to an arbitrary placed "source" to a "detector" on the circumference, including those which go through an arbitrary number of full roundabouts. This problem allows us to introduce the concept of quantization of angular momentum.

\section{The Bohr atomic model revisited}

At the end of the sequence, we introduce Bohr's model of the atom as seen from the point of view of the sum over paths approach, and discuss its limitations. The model is based on the assumption that the electron is confined to take all paths on a circular orbit around the proton, a circular orbit which must be permitted by classical mechanics. Thus, the same result obtained in the previous section can be applied, and the Bohr energy levels and allowed orbit radiuses are found as a result. The limitations of the models which are discussed are (1) the model is two dimensional, and (2) it does not take into account all possible paths, but only those fictitiously confined to a circumference. The orbital model is then discussed qualitatively, showing that the Bohr radius corresponds to the maximum of the $1 s$ orbital wave function of hydrogen; this allows us to conclude that, in principle, the peaks of such a wave function derive from a constructive interference condition, similar in principle to the one found in the semiclassical model.

\section{APPENDIX C: RELEVANT EXERCISES FROM THE ASSESSMENT TEST FOR SCHOOL GRADING}

(1) An electron beam with de Broglie wavelength $\lambda=$ $2.0 \mathrm{~m} \times 10^{-7} \mathrm{~m}$ is directed towards an unsurmountable obstacle with two slits, through which electrons can pass, which are $d=1.0 \mathrm{~mm}$ apart. The electrons are then detected on a screen which is $D=5.0 \mathrm{~m}$ distant from the slits.

(a) Considering the slits as pointlike, calculate the distance between two successive maxima of the observed interference pattern, using the approximations of Fraunhofer and of small angles.

(b) Considering now slits with very small but finite width (for example, $a=1.0 \mathrm{~m} \times 10^{-5} \mathrm{~m}$ ) describe qualitatively what would be observed on the screen if, on one of the slits, a detector is placed, capable of revealing the passage of the electron without disturbing it. Precise calculations are not necessary.

(2) A Michelson interferometer is formed by two mirrors and a beam splitter (with 50/50 reflection to transmission probability) set up as in Fig. 10.

(a) Identify the possible paths between the source $(S)$ and the detector $(D)$.

(b) If the traits P-M1 and P-M2 are exactly equal in length, calculate the expected probability of finding a photon at $D$.

(c) If the source emits green photons with wavelength $\lambda=430 \mathrm{~nm}$ find the minimum variation in the distance P-M2 needed to obtain a maximum probability of detecting a photon at $D$.

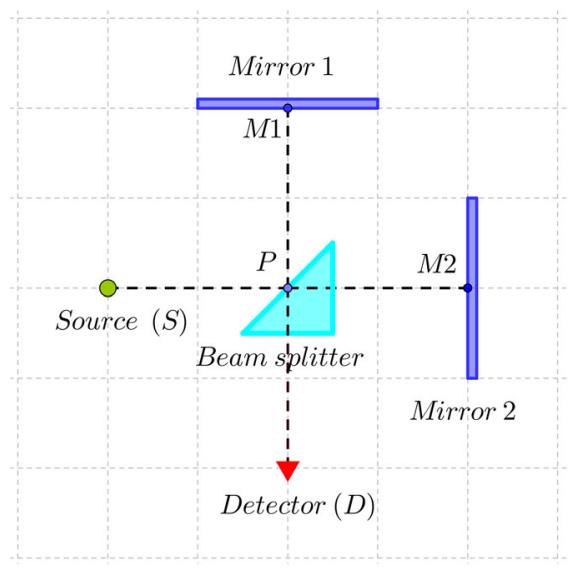

FIG. 10. The Michelson interferometer. 
[1] R. P. Feynman, Space-time approach to non-relativistic quantum mechanics, Rev. Mod. Phys. 20, 367 (1948).

[2] R. P. Feynman, QED: The Strange Theory of Light and Matter (Princeton University Press, Princeton, NJ, 1985).

[3] R. P. Feynman and A. R. Hibbs, Quantum Mechanics and Path Integrals (MacGraw Hill, New York, NY, 1965).

[4] E. F. Taylor, S. Vokos, J. M. O'Meara, and N. S. Thornber, Teaching Feynman's sum-over-paths quantum theory, Comput. Phys. 12, 190 (1998).

[5] D. F. Styer, The Strange World of Quantum Mechanics (Cambridge University Press, New York, 2000).

[6] G. Rinaudo, Il metodo di Feynman della somma su molti cammini per l'introduzione della Meccanica Quantistica, Formazione a Distanza Degli Insegnanti all'Innovazione Didattica in Fisica Moderna e Orientamento, edited by M. Michelini (LithoStampa, Udine, Italy, 2010).

[7] M. Malgieri, P. Onorato, and A. De Ambrosis, Teaching quantum physics by the sum over paths approach and GeoGebra simulations, Eur. J. Phys. 35, 055024 (2014).

[8] M. de los Àngeles Fanaro and M. R. Otero, Basics Quantum Mechanics teaching in secondary school: One conceptual structure based on Paths Integrals Method, Latin-Am. J. Phys. Educ. 2, 103 (2008).

[9] Advancing Physics AS, edited by J. Ogborn and M. Whitehouse (Institute of Physics, Bristol, United Kingdom, 2000).

[10] K. Dobson, I. Lawrence, and P. Britton, The A to B of quantum physics, Phys. Educ. 35, 400 (2000).

[11] G. Ireson, The quantum understanding of pre-university physics students, Phys. Educ. 35, 15 (2000).

[12] H. Fischler and M. Lichtfeldt, Modern physics and students' conceptions, Int. J. Sci. Educ. 14, 181 (1992).

[13] K. Mannila, I. T. Koponen, and J. A. Niskanen, Building a picture of students' conceptions of wave-and particle-like properties of quantum entities., Eur. J. Phys. 23, 45 (2002).

[14] G. Papaphotis and G. Tsaparlis, Conceptual versus algorithmic learning in high school chemistry: the case of basic quantum chemical concepts. Part 1. Statistical analysis of a quantitative study, Chem. Educ. Res. Pract. 9, 323 (2008).

[15] I. D. Johnston, K. Crawford, and P. R. Fletcher, Student difficulties in learning quantum mechanics, Int. J. Sci. Educ. 20, 427 (1998).

[16] B. A. Thacker, A study of the nature of students' models of microscopic processes in the context of modern physics experiments, Am. J. Phys. 71, 599 (2003).

[17] R. V. Olsen, Introducing quantum mechanics in the upper secondary school: a study in Norway, Int. J. Sci. Educ. 24, 565 (2002).

[18] A. Mashhadi and B. Woolnough, Insights into students' understanding of quantum physics: visualizing quantum entities, Eur. J. Phys. 20, 511 (1999).

[19] M. Ayene, J. Kriek, and B. Damtie, Wave-particle duality and uncertainty principle: Phenomenographic categories of description of tertiary physics students' depictions, Phys. Rev. ST Phys. Educ. Res. 7, 020113 (2011).

[20] J. Petri and H. Niedderer, A learning pathway in high school level quantum atomic physics, Int. J. Sci. Educ. 20, 1075 (1998).

[21] B. S. Ambrose, P. S. Shaffer, R. N. Steinberg, and L. C. McDermott, An investigation of student understanding of single-slit diffraction and double-slit interference, Am. J. Phys. 67, 146 (1999).

[22] R. Müller and H. Wiesner, Students' conceptions of quantum physics, in Research in Teaching and Learning Quantum Mechanics, Papers presented at the annual meeting of the National Association for Research in Science Teaching March, 1999, edited by D. Zollman (1999) p. 20,https://web.phys.ksu.edu/papers/narst/ QM_papers.pdf.

[23] R. Müller and H. Wiesner, Teaching quantum mechanics on an introductory level, Am. J. Phys. 70, 200 (2002).

[24] J. Falk, Ph.D. thesis, Uppsala University 2007.

[25] G. Kalkanis, P. Hadzidaki, and D. Stavrou, An instructional model for a radical conceptual change towards quantum mechanics concepts, Sci. Educ. 87, 257 (2003).

[26] G. Tsaparlis and G. Papaphotis, High school Students' Conceptual Difficulties and Attempts at Conceptual Change: The case of basic quantum chemical concepts, Int. J. Sci. Educ. 31, 895 (2009).

[27] S. Vosniadou, X. Vamvakoussi, and I. Skopeliti, The framework theory approach to the problem of conceptual change, in International Handbook of Research on Conceptual Change, edited by S. Vosniadou (Routledge, Oxford, 2008), pp 3-34.

[28] M. Malgieri, Ph. D. thesis, University of Pavia, ISBN 97888-95767-92-5, 2015.

[29] S. Vosniadou, C. Ioannides, A. Dimitrakopoulou, and E. Papademetriou, Designing learning environments to promote conceptual change in science, Learn. Instr. 11, 381 (2001).

[30] S. Vosniadou and I. Skopeliti, Conceptual change from the framework theory side of the fence, Sci. Educ. 23, 1427 (2014).

[31] H. S. Lee, M. C. Linn, K. Varma, and O. L. Liu, How do technology-enhanced inquiry science units impact classroom learning?, J. Res. Sci. Teach. 47, 71 (2010).

[32] O. L. Liu, H. S. Lee, and M. C. Linn, Measuring knowledge integration: Validation of four year assessments, J. Res. Sci. Teach. 48, 1079 (2011).

[33] M. C. Linn, The knowledge integration perspective on learning and instruction, in The Cambridge Handbook of the Learning Sciences, edited by R. K. Sawyer (Cambridge University Press, New York, 2006), pp. 243-264.

[34] D. B. Clark and M. C. Linn, The knowledge integration perspective: Connections across research and education, in International Handbook of Research on Conceptual Change, edited by S. Vosniadou (Routledge, Oxford, 2013), pp. 520-538.

[35] O. L. Liu, H.S. Lee, C. Hofstetter, and M. C. Linn, Assessing knowledge integration in science: Construct, measures, and evidence, Educ. Assess. 13, 33 (2008).

[36] M. Schneider and I. Hardy, Profiles of inconsistent knowledge in children's pathways of conceptual change, Dev. Psychol. 49, 1639 (2013).

[37] K. Southard, T. Wince, S. Meddleton, and M. S. Bolger, Features of knowledge building in biology: Understanding undergraduate students' ideas about molecular mechanisms, CBE-Life Sci. Educ. 15, ar7 (2016).

[38] M. Malgieri, P. Onorato, and A. De Ambrosis, Design and Refinement of an introductory teaching-learning sequence 
in quantum physics, in Electronic Proceedings of the ESERA 2015 Conference. Science Education Research: Engaging Learners for a Sustainable Future, edited by J. Lavonen, K. Juuti, J. Lampiselkä, A. Uitto, and K. Hahl (University of Helsinki, Helsinki, 2016), ISBN 978-95151-1541-6.

[39] X. Y. Zhou, L. J. Wang, and L. Mandel, Induced Coherence and Indistinguishability in Optical Interference, Phys. Rev. Lett. 67, 318 (1991).

[40] M. Malgieri, P. Onorato, and A. De Ambrosis, A learning path on quantum physics including simulations, low cost experiments, online resources., in Proceedings of the 20th International Conference on Multimedia in Physics Teaching and Learning, edited by L.-J. Thoms and R. Girwidz (European Physical Society, Mulhouse, 2016), ISBN 978-2-914771-94-8.

[41] J. D. Cutnell and K. W. Johnson, Fisica blu, edited by C. Romeni (Zanichelli, Bologna, 2012).

[42] M. Malgieri, P. Onorato, and A. De Ambrosis, A sumover-paths approach to one-dimensional time-independent quantum systems, Am. J. Phys. 84, 678 (2016).

[43] P. Onorato Low-dimensional nanostructures and a semiclassical approach for teaching Feynman's sum-over-paths quantum theory, Eur. J. Phys. 32, 259 (2011).

[44] See Supplemental Material at http://link.aps.org/ supplemental/10.1103/PhysRevPhysEducRes.13.010101 for a more extended description of the sequence steps.

[45] O. Levrini, P. Fantini, G. Tasquier, B. Pecori, and M. Levin, Defining and operationalizing appropriation for science learning, J. Learn. Sci. 24, 93 (2015).

[46] F. Zhou and T. Cloninger, Computer-based experiment for determining Planck's constant using LEDs, Phys. Teach. 46, 413 (2008).

[47] P. Onorato, M. Malgieri, and A. De Ambrosis, Measuring the hydrogen Balmer series and Rydberg's constant with a homemade spectrophotometer, Eur. J. Phys. 36, 058001 (2015).

[48] O. Levrini, A. De Ambrosis, L. Branchetti, M. Malgieri, and G. Tasquier, Applying the construct of "appropriation" to design and analyse teaching experiences on quantum physics (to be published).

[49] P. G. Merli, G. F. Missiroli, and G. Pozzi, On the statistical aspect of electron interference phenomena, Am. J. Phys. 44, 306 (1976).
[50] M. G. Hennessey, Metacognitive aspects of students' reflective discourse: Implications for intentional conceptual change teaching and learning, in Intentional Conceptual Change, edited by G. M. Sinatra and P. R. Pintrich (Routledge, London, 2003).

[51] M. Chi, Conceptual Change within and across Ontological Categories: Examples from Learning and Discovery in Science, in Cognitive Models of Science: Minnesota Studies in the Philosophy of Science Vol. 15, edited by R. Giere (University of Minnesota Press, Minneapolis, 1992), pp. 129-186.

[52] J. Ogborn, A First Introduction to Quantum Behavior, edited by E. In van den Berg, T. Ellermeijer, and T. Slooten Proceedings of the GIREP Conference 2006: Modelling in Physics and Physics Education (University of Amsterdam, Alphen aan den Rijn, Netherlands, 2006).

[53] U. Amaldi, Corso di Fisica, 5th ed. (Zanichelli, Bologna, 2003), Vol. 3.

[54] J. F. Roch, F. Treussart, and P. Grangier, Single photon interference with a Fresnel biprism. [Video file]. Retrieved from http://www.physique.ens-cachan.fr/old/franges photon/interference.htm (2005).

[55] A. Stefanel, Impostazioni e percorsi per l'insegnamento della meccanica quantistica nella scuola secondaria, Giornale di Fisica 49, 15 (2008).

[56] P. L. Muiño, Introducing the uncertainty principle using diffraction of light waves, J. Chem. Educ. 77, 1025 (2000).

[57] S. Frabboni, G. C. Gazzadi, V. Grillo, and G. Pozzi, Elastic and inelastic electrons in the double-slit experiment: A variant of Feynman's which-way set-up, Ultramicroscopy 154, 49 (2015).

[58] A. Zeilinger, C. G. Shull, W. Treimer, and W. Mampe, Single- and double-slit diffraction of neutrons, Rev. Mod. Phys. 60, 1067 (1988).

[59] M. Arndt, O. Nairz, J. Vos-Andreae, C. Keller, G. Van der Zouw, and A. Zeilinger, Wave-particle duality of C60 molecules, Nature (London) 401, 680 (1999).

[60] S. Vokos, P. S. Shaffer, B. S. Ambrose, and L. C. McDermott, Student understanding of the wave nature of matter: Diffraction and interference of particles, Am. J. Phys. 68, S42 (2000).

[61] M. Malgieri, A. Tenni, P. Onorato, and A. De Ambrosis, What Feynman could not yet use: The generalised HongOu-Mandel experiment to improve the QED explanation of the Pauli exclusion principle, Phys. Educ. 51, 055002 (2016). 